\title{
نموذج مقترح لتصنيف ومعدلات الخدمات الإدارية بالمدن المصرية
}

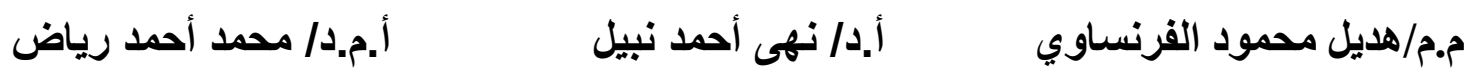

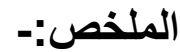

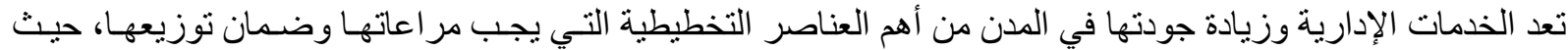

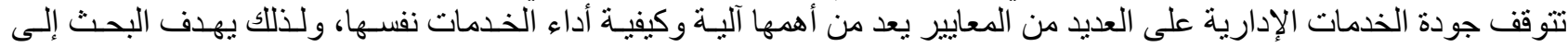

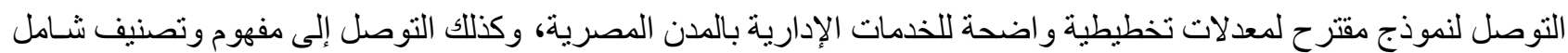

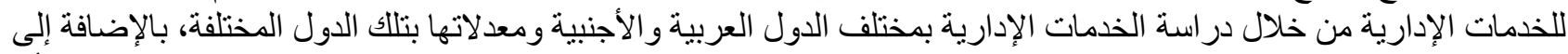

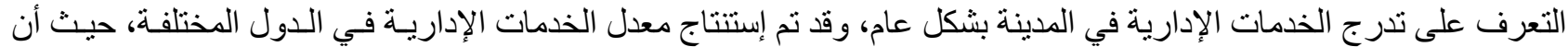

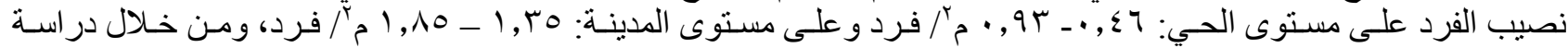

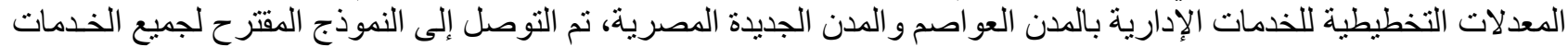

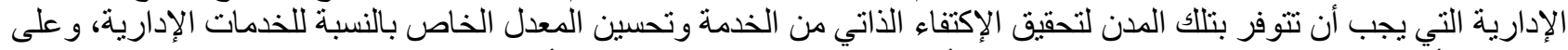

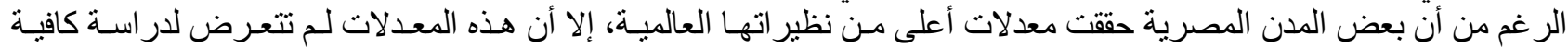

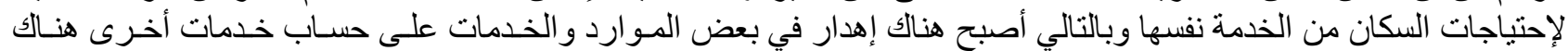
قصور فيها.

الكلمات الدلالية: الخدمات الإدارية، المعدلات التخطيطية، الددن العو اصم، الددن الجديدة.

\section{Abstract:}

Administrative services and improving their quality in cities are two of the most significant planning factors that must be considered and distributed. Where the quality of administrative services is influenced by many factors, the most significant of which is the mechanism and how the services are delivered. Therefore, the research aims to reach a proposed model for administrative service planning rates in Egyptian cities. In addition to developing a complete concept and classification of administrative services by researching administrative services in various Arab and international nations, as well as their rates in those countries. Besides identifying the hierarchy of administrative services in the city in general. The average of administrative services in different countries was concluded, given the per capita share at the district level: $0,46-0,93 \mathrm{~m}^{2} /$ person, and at the city level: $1,35-1,85 \mathrm{~m}^{2} /$ person. By exploring the planning rates for administrative services in Egyptian capital cities and new cities, the proposed model for all administrative services that must be available in those cities to achieve self-sufficiency in service and improve the special rate for administrative services was developed. Although some Egyptian cities have reached higher rates than their worldwide equivalents, these rates have not been fully examined for the population's demands for the same service, and as a result, certain resources and services have been wasted at the loss of other services that have problems.

Key Words: Administrative Services, Planning Rates, Capital Cities, New Cities.

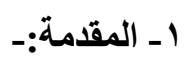

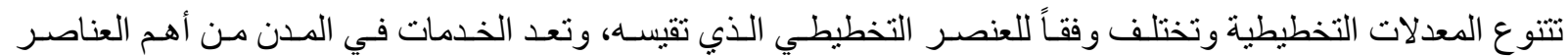

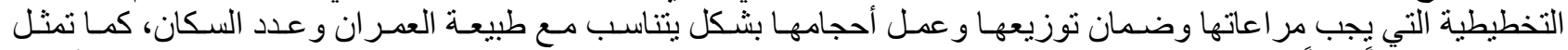

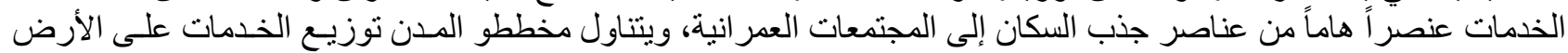

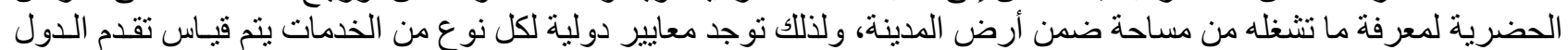
وتخلفها وفق تلك المعايير.

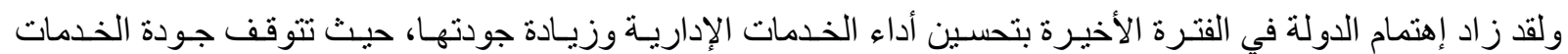

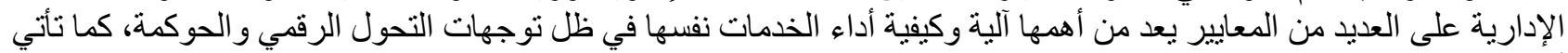

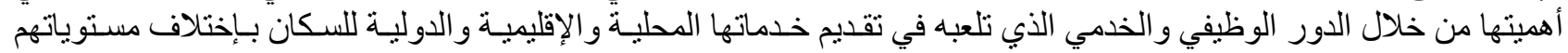




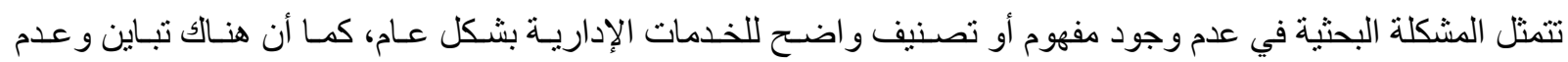

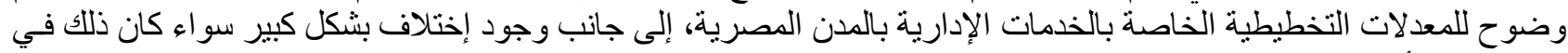

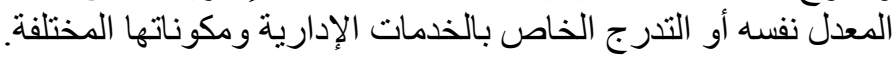

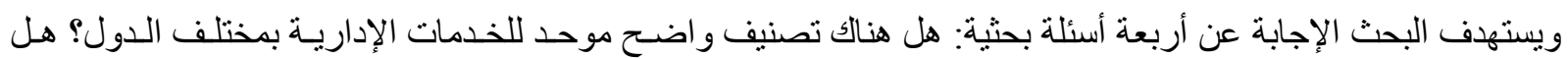

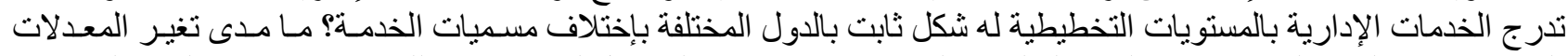

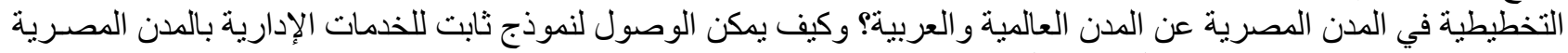
يمكن تطبيقه عند تصميم وتخطيط المدرية الخدمات العامةن؟ العبة

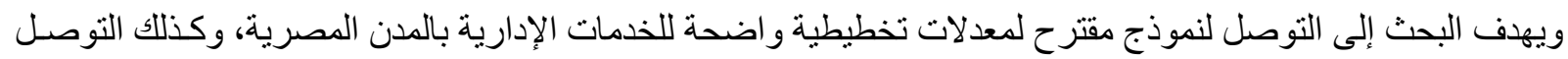

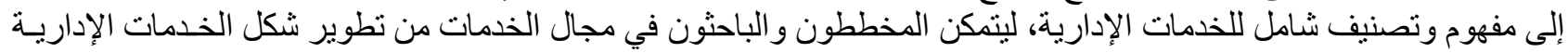
ومو اكبتها مع معطيات المدن في العصر الحديث. كما بتضمن البحث ثلاثة محاور أساسية:

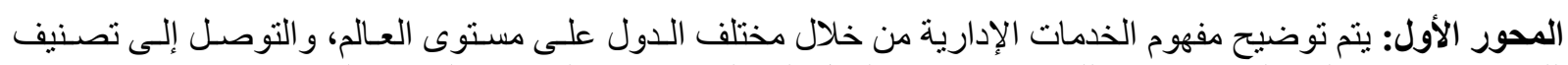

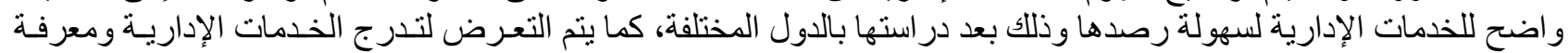
مكوناتها بالمستو يات التخطيطية المختلفة في المدينة.

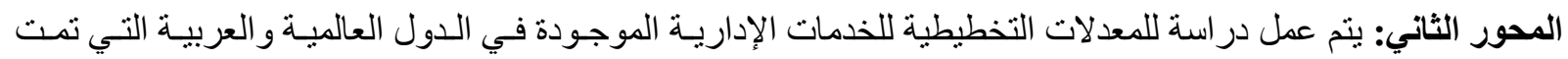

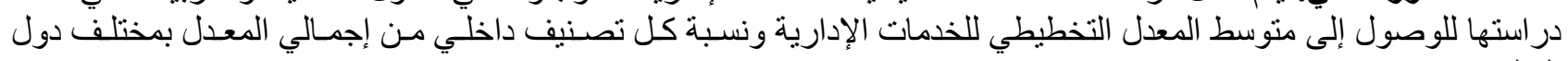

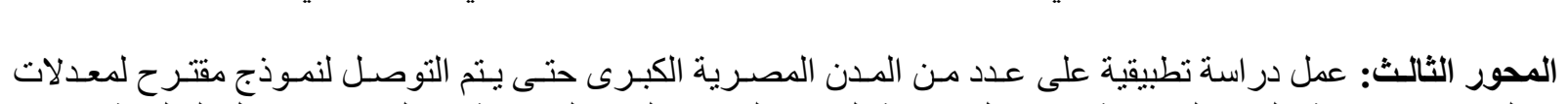
العالم.

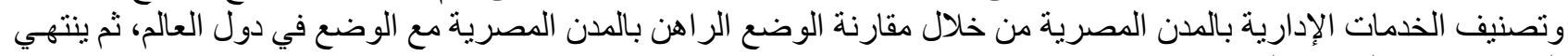
البحث بعرض النتائج و التوصيات.

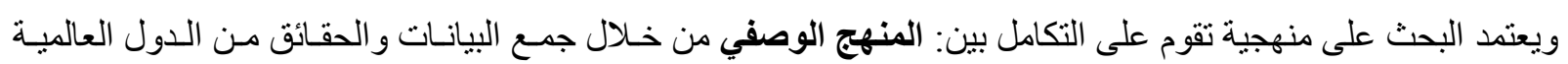

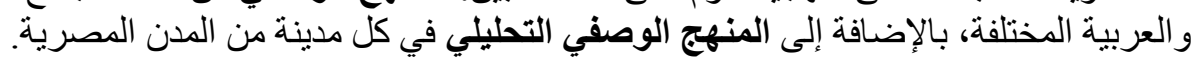

\section{r ا الخدمات الإدارية بمختلف الدول العربية والأجنبية}

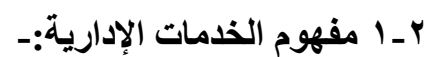

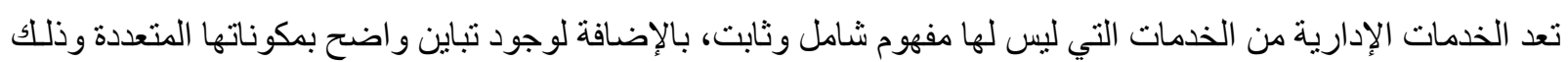

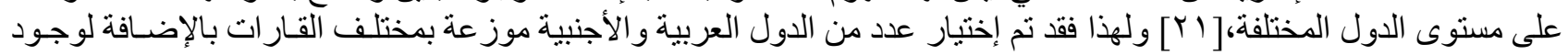
تتوع في المستوى الإقتصادي بتللك الدول، للوصول إلى فهم شامل لمفهوم الخدمات الإداريـة وكذللك تصنيفاتها المختلفـة فيمـا بعد، وفيما يلّي عرض لتلك المبتوى المفاهيم:-

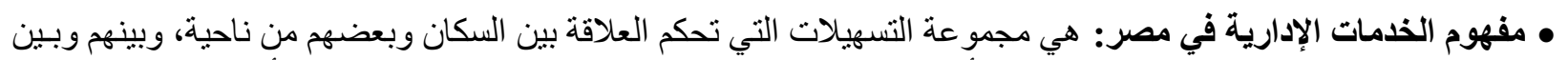

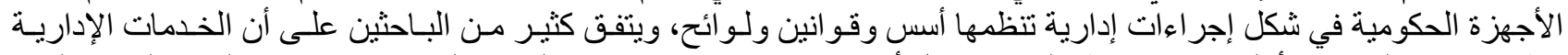

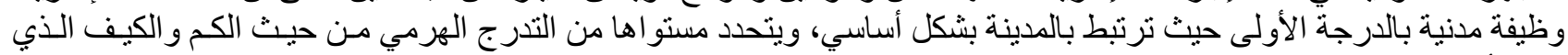

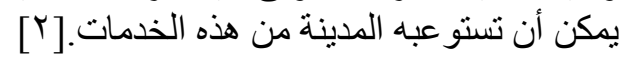

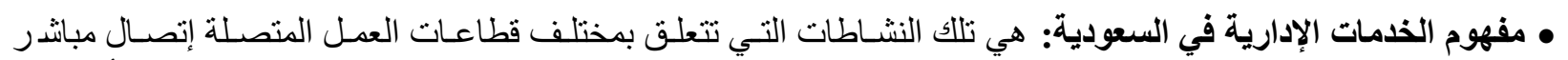

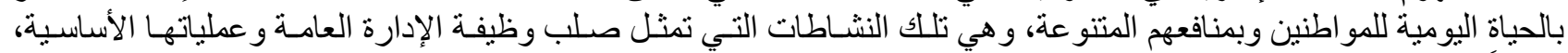

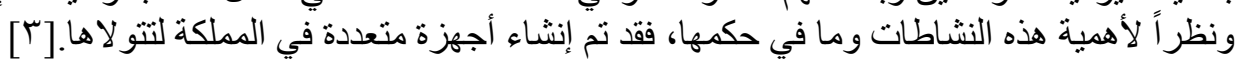

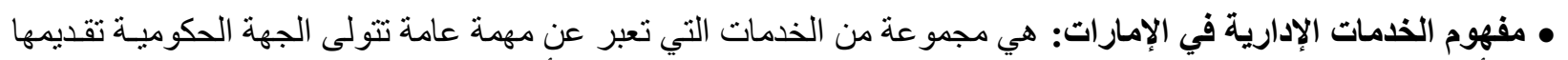

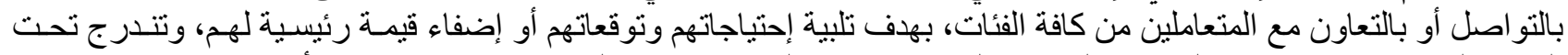

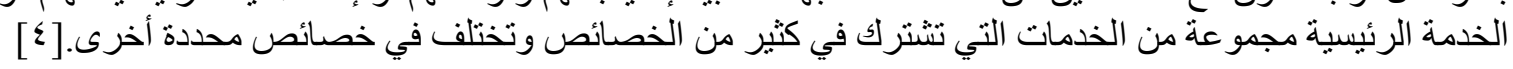




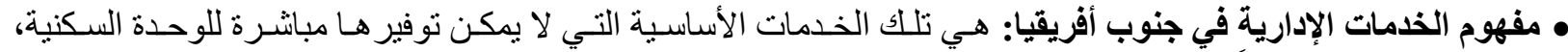

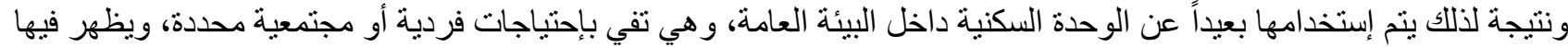

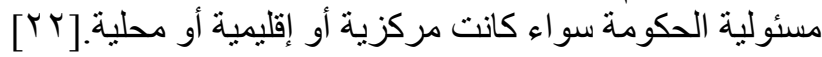

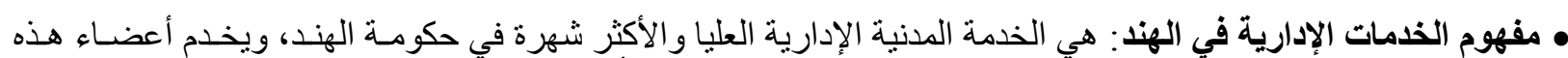

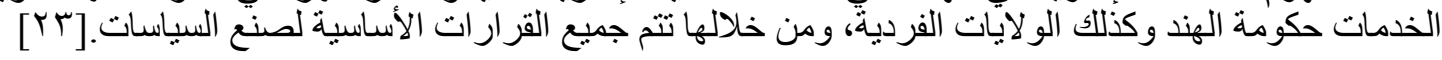

• مفهوم الخدمات الإدارية في الصين: هي مجمو عة المر افق ذات الأهميـة المحليـة والتي يتم إستخدامها مـن السكان بشكل

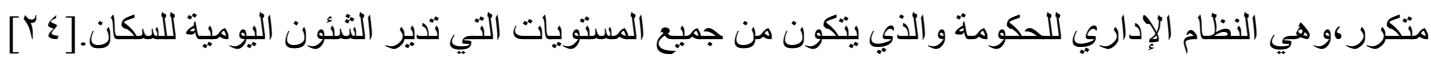

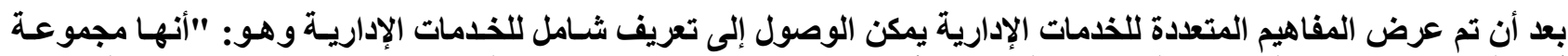

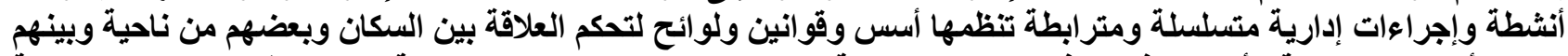

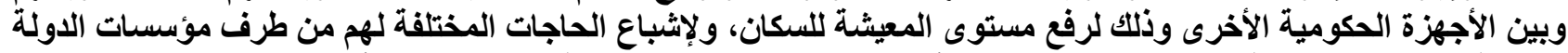

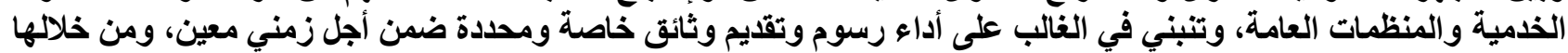

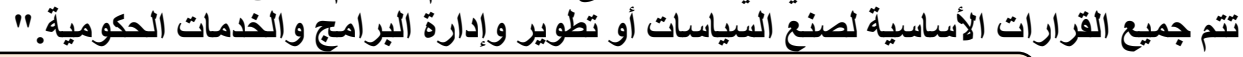

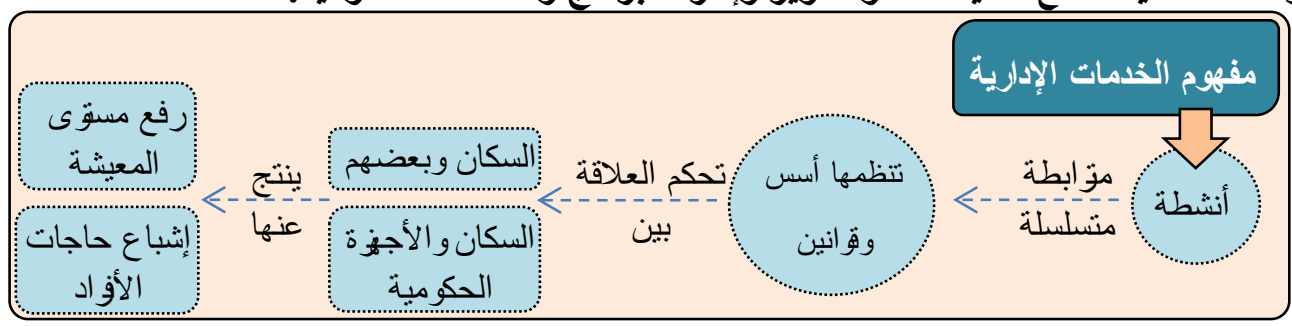

شكل ( ) يوضح المفهوم المبسط للخدمات الإدارية

المصدر: الباحثة

يوجد تباين في شكل ومحتوى الخدمات الإدارية بالنسبة للادول المختلفة، فهناك دول تعتبر الخدمات الخاصة بالمباه والكات والكهرباء

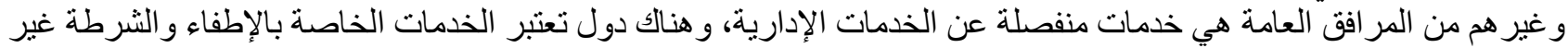

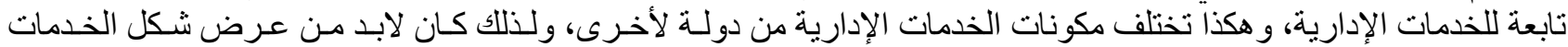

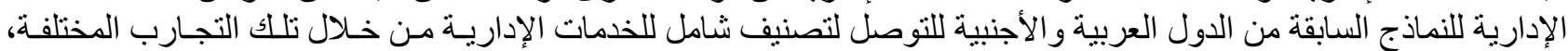

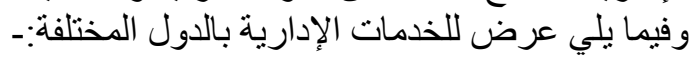

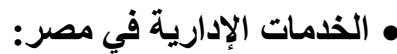

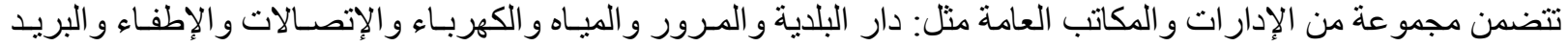

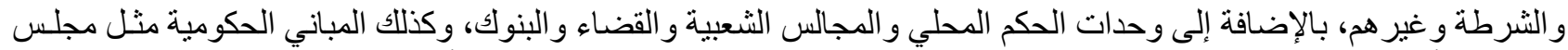

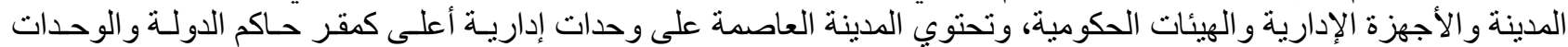

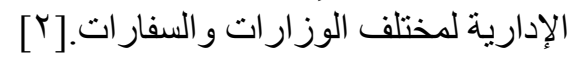

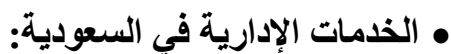

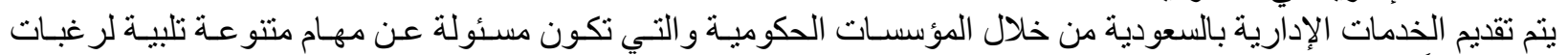

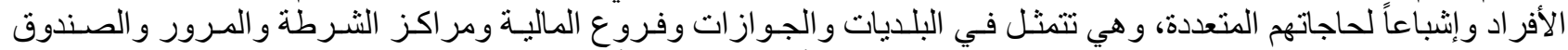

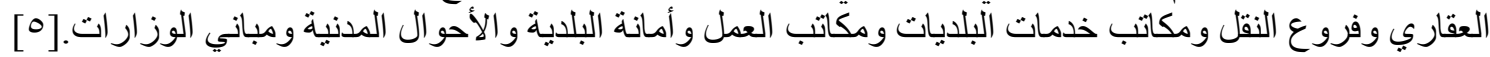

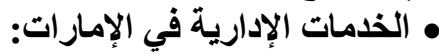

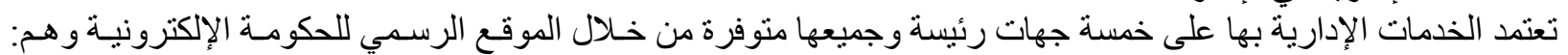

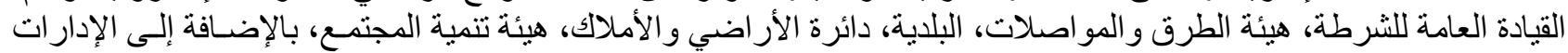

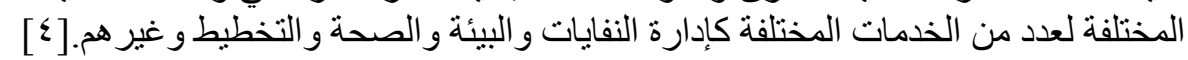
• 1 الخدمات الإدارية في جنوب أفريقيا:

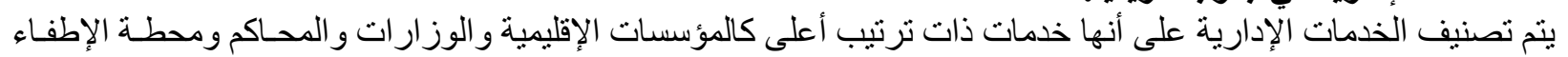

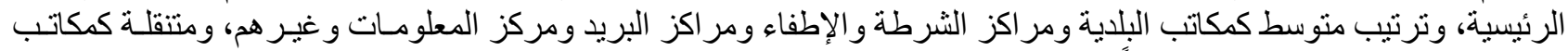

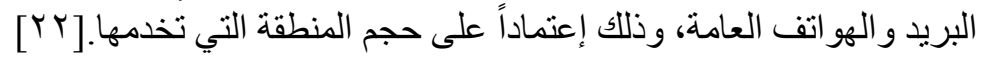




\section{• ت الخدمات الإدارية في الإند:}

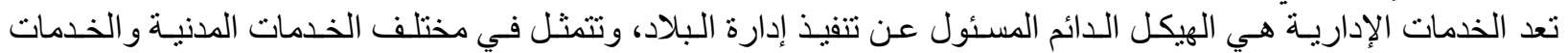

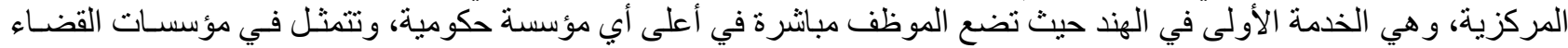

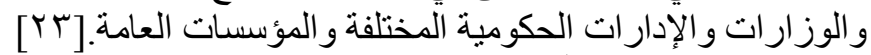

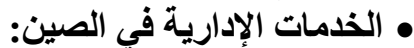

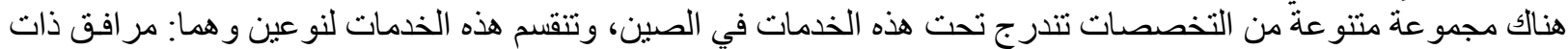

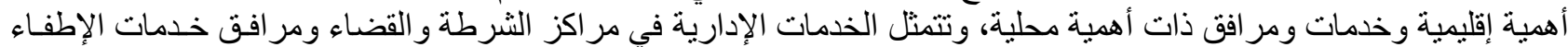

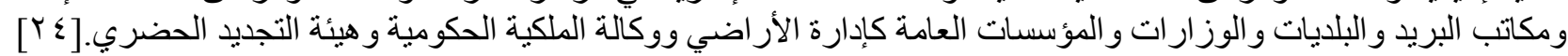

بعد أن تم توضيح الخدمات الإداريـة وأنواعها داخل العديـ مـن الدول العربيـة والأجنبيـة، يمكن الوصـول إلى تصنيف شـامل للخدمات الإدارية وذلك كما يأتي:- الإدارية

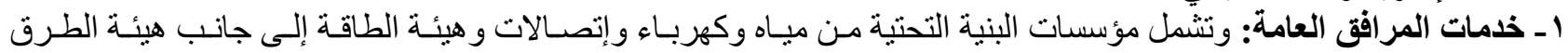

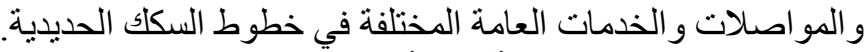

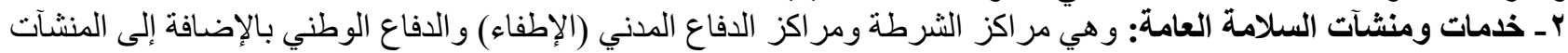
العسكرية ذات الإستخدام الخاص.

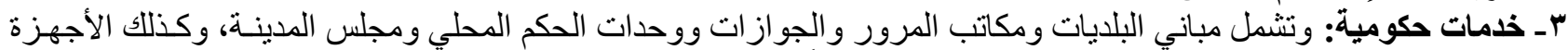
الإدارية و الهيئات و الجهات الحكومية و الجمارك و البريد، و أيضاً هيئة تنمية المجتمع و الوحدات الئات الإدارية الإقليمية.

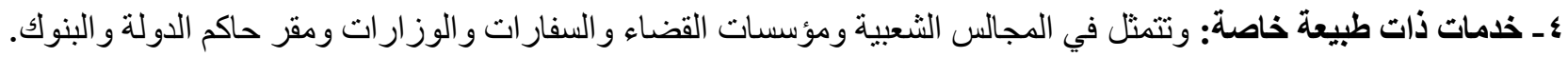

\section{تصنيف الخدمات الإدارية}

\begin{tabular}{|c|c|c|c|c|c|}
\hline \multirow{2}{*}{\multicolumn{2}{|c|}{ خدمات ذات طبيعة خاصة }} & & & & \\
\hline & & \multicolumn{2}{|c|}{ خدمات حكومية } & |خدمات ومنشآت السلامة & خدمات العوافق العامة \\
\hline \multicolumn{2}{|c|}{ المجالس الثُعبية } & مجلس المدينة & البلدياتي & مراكز الثرطة & مؤسسات البنية التحتية \\
\hline \multicolumn{2}{|c|}{ مؤسسات القضاء } & اليويد & المورور & الدفاع المدني & هيئة الطُقو المواصلات \\
\hline \multicolumn{2}{|c|}{ الوزر ات و السفر ات } & الأجزة الإدرية & الجمرك & الدفاع الوطني & هيئة السكك الحديدية \\
\hline مقر حاكم & البنوك & الوحدات الإدلرية & العقلية المتلكات & المنشآت العسكية & هيئة الطاقة \\
\hline
\end{tabular}

شكل (r): تصنيف الخدمات الإدارية ـ المصدر: الباحثة

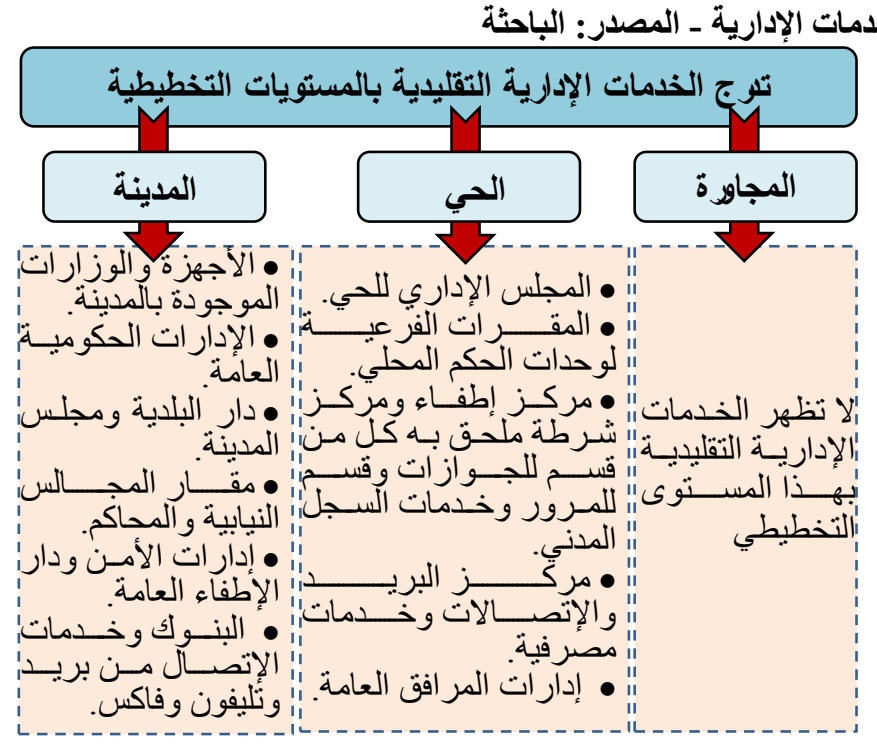

شكل (r): تدرج الخدمات الإدارية بالمستويات التخطيطية

المصدر: Bauby, Pierre \& Others, "Public Services in the European Union \& in the 27 Member States", The European Comission, 2010, p.82. r r ب ت برج الخدمات الإدارية:-

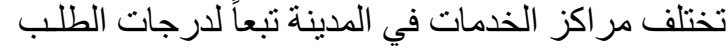

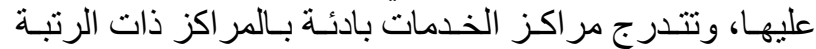

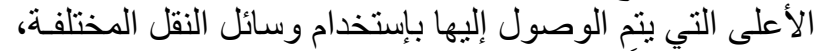

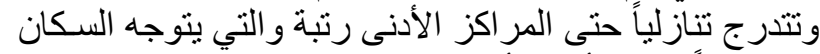

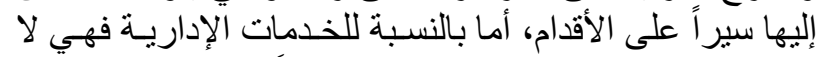

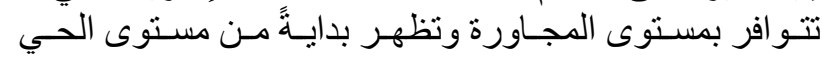

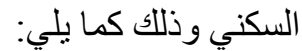

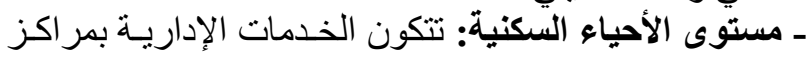

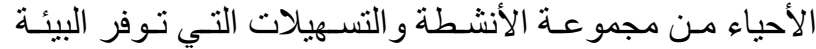

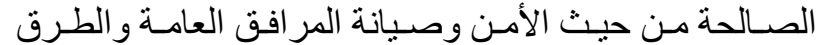

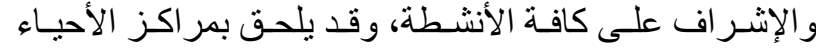

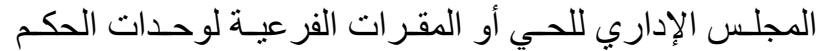

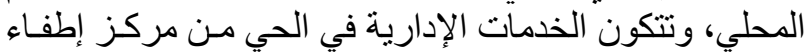

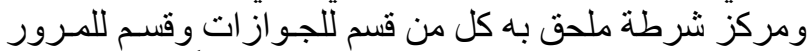

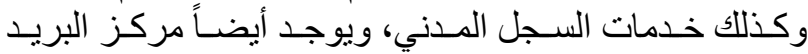

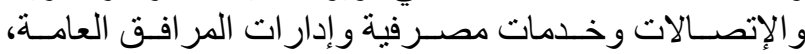




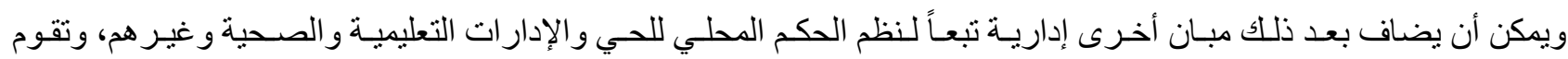

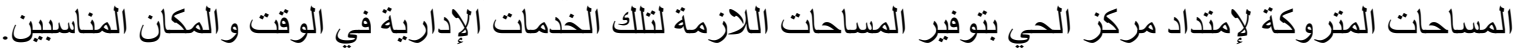

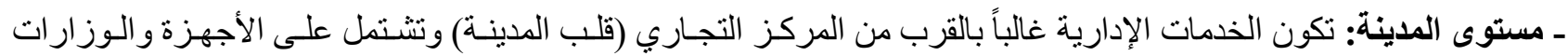

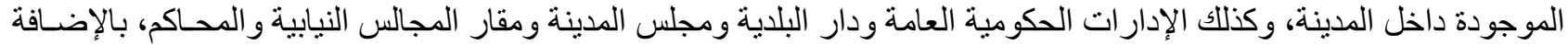

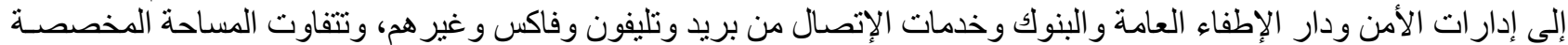

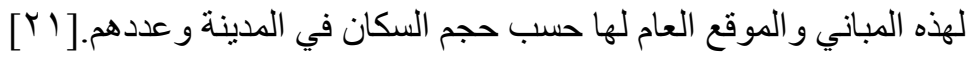

r- بـ المعدلات التخطيطية للخدمات الإدارية بالدول المختلفة:-

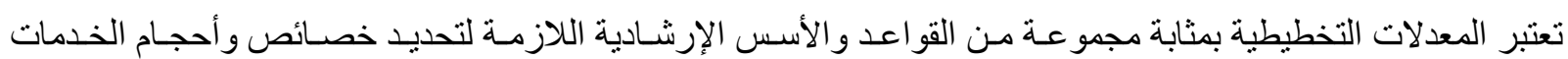

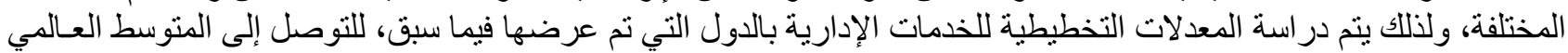

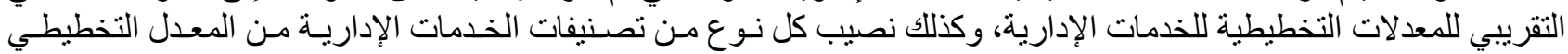

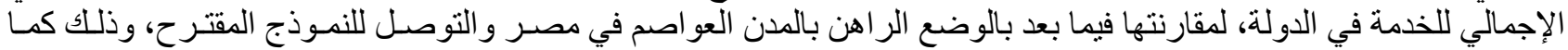

جدول (1): المعدلات التخطيطية للخدمات الإدارية بالدول المختلفة

\begin{tabular}{|c|c|c|c|c|c|c|c|}
\hline \multicolumn{6}{|c|}{ المستوى التخطيطى } & \multirow[b]{3}{*}{ نوع الخدمة } & \multirow[b]{3}{*}{ الادراسنة } \\
\hline \multicolumn{3}{|c|}{ المدينة } & \multicolumn{3}{|c|}{ الحي } & & \\
\hline النسبة & المسيبة (الفرد من منرد) & (ألف المدومين & | النسبة & |المساحة (مّبّة الفرد) & (ألف نسمدمين السكان & & \\
\hline$\%$ & $\cdot, Y \wedge$ & \multirow{4}{*}{ ro. - 1 . } & $\% 11$ &., .0 & \multirow{4}{*}{$0 .-r$. } & خدمات مرافق عامة & \multirow{4}{*}{ 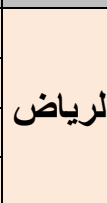 } \\
\hline$\% 1 \wedge$ & $\cdot, Y \Sigma$ & & $\% Y V$ & $\cdot, 1 \pi$ & & خدمات السلامة العامة & \\
\hline$\% \varepsilon r$ &., 01 & & $\%$ \% & $\cdot, 17$ & & الخدمات الحكومية & \\
\hline$\%$ & $\cdot, \mathrm{YA}$ & & $\%$ \% & $\cdot, 1 T$ & & خلمات ذات طبيعة خاصة & \\
\hline \multicolumn{3}{|c|}{ على مستوى المدينة rv } & \multicolumn{3}{|c|}{ على مستوى الحي \َ ؛ , . } & \multicolumn{2}{|c|}{ متوسط معدل الخدمات الإدارية } \\
\hline$\% 19$ & $\cdot, Y_{O}$ & \multirow{4}{*}{$r \ldots-0}$. & $\% 17$ &., .9 & \multirow{4}{*}{$r \cdot-r \cdot$} & خدمات مرافق عامة & \multirow{4}{*}{ دبي } \\
\hline$\% 1 \leq$ & $\cdot, Y$ & & $\%$ \% &., 10 & & خدمات السلامةٌة العامة & \\
\hline$\%$ \% & $\cdot .74$ & & $\%$ \% & $\cdot, Y Y$ & & الخدمات الحكومية & \\
\hline$\% Y_{1}$ & $\cdot, Y A$ & & $\% Y_{1}$ & $\cdot, 1 Y$ & & خدمات ذات طبيعة خاصة & \\
\hline \multicolumn{3}{|c|}{ على مستوى المدينة هر, 1, } & \multicolumn{3}{|c|}{ على مستوى الحي ^ه, • } & \multicolumn{2}{|c|}{ متوسط معدل الخدمات الإدارية } \\
\hline$\%$ & $\cdot, Y$ & \multirow{4}{*}{$1 \cdots-7}$. & $\% \wedge$ & $\cdot, \cdot V$ & \multirow{4}{*}{$7 \cdot-11$} & | خدمات مرافق عامة & \multirow{4}{*}{ أفريقيا } \\
\hline$\% 17$ & $\cdot$, YO & & $\% \curlyvee \wedge$ & $\cdot, Y T$ & & خدمات السلامة العامـة & \\
\hline$\% \leqslant \wedge$ & $\cdot, \vee \vee 0$ & & $\%$ \%० & $\cdot, \S)$ & & الخدمات الحكومية & \\
\hline$\%$ \% & $\cdot, \mu q$ & & $\% 19$ & $\cdot, 11$ & & خدمات ذات طبيعة خاصة & \\
\hline \multicolumn{3}{|c|}{ على مستوى المدينة ^ه, , } & \multicolumn{3}{|c|}{ على مستوى الحي 19, . } & \multicolumn{2}{|c|}{ متوسط معدل الخدمات الإدارية } \\
\hline$\% 17$ & $\cdot r$ & \multirow{4}{*}{$-1 \ldots$} & $\% 9$ & $\cdot, \cdot \wedge$ & \multirow{4}{*}{$q \cdot-\leqslant$. } & خدمات مرافق عامة & \multirow{4}{*}{ الهند الهـ } \\
\hline$\% 1 V$ & $\cdot, \pi r$ & & $\% r$ & $\cdot, r$ & & خدمات السلامةة العامة & \\
\hline$\% \varepsilon r$ & $\cdot, \vee \wedge$ & & $\% \leqslant r$ & $\cdot, \varepsilon$ & & الخدمات الحكومية & \\
\hline$\%$ \% & $\cdot, \leqslant 0$ & & $\% 1 \mathrm{~V}$ & $\cdot, 17$ & & خدمات ذات طبيعة خاصة & \\
\hline \multicolumn{3}{|c|}{ على مستوى المدينة هر, 1 } & \multicolumn{3}{|c|}{ على مستوى الحي بـ9, . } & \multicolumn{2}{|c|}{ متوسط معدل الخدمات الإدارية } \\
\hline$\% \mathrm{~V}$ & $\cdot \Gamma$ & \multirow{4}{*}{$7 T \cdot-r \ldots$} & $\% 1 r$ & $\cdot, 1$ & & | خدمات مرافق عامة & \multirow{4}{*}{ 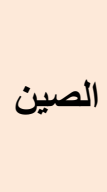 } \\
\hline$\% \curlywedge \wedge$ & $\cdot, \Upsilon \wedge$ & & $\% 1$ & $\cdot, r V$ & & خدمات السلامة العامة & \\
\hline$\% \leqslant 0$ & $\cdot, \mathrm{TV}$ & & $\% \varepsilon r$ & $\cdot, \Gamma \wedge$ & & الخدمات الحكومية & \\
\hline$\%(Y)$ & $\cdot, \Gamma$ & & $\% 1 \leq$ & $\cdot, 1 \mu$ & & خدمات ذات طبيعة خاصة & \\
\hline & توى المدينة إن & على & & توى الحي AV, • & & لـ معدل الخدمات الإدارية & متوسط \\
\hline 1 , & ل دينة هr, & على ه & & لحي 1 \&, - - & على مد & على معدل الذدمات الإلدارية & متوسط \\
\hline
\end{tabular}

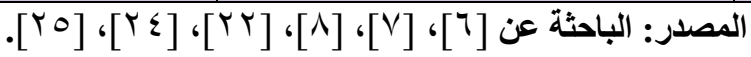




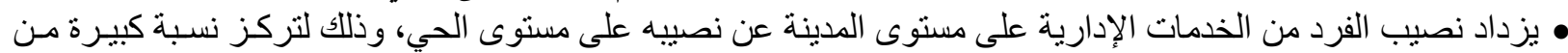

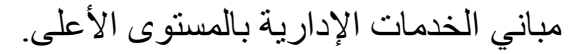

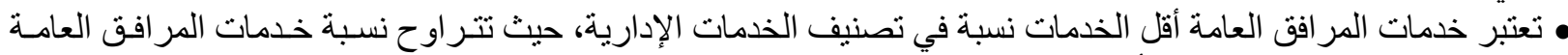

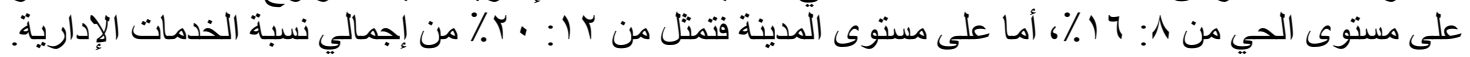

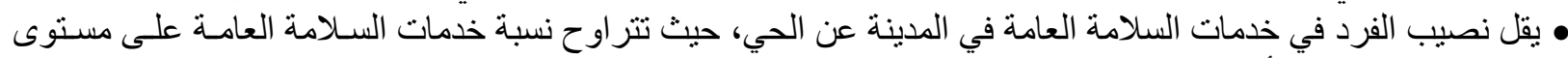

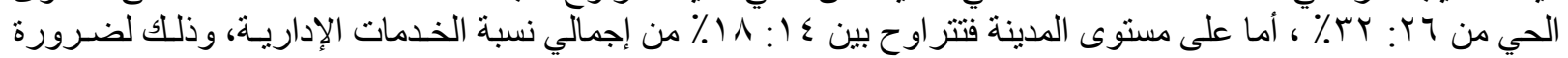

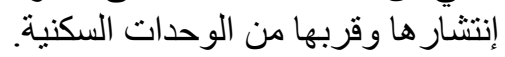

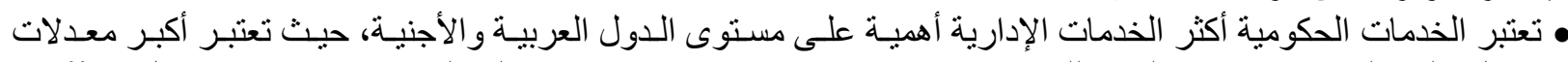

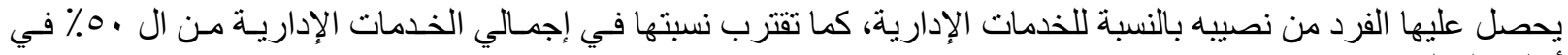

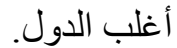

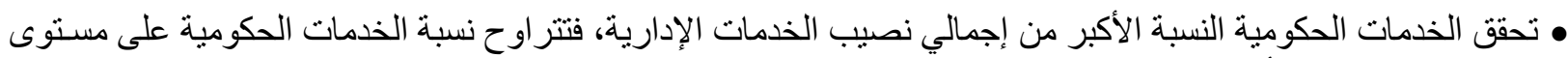

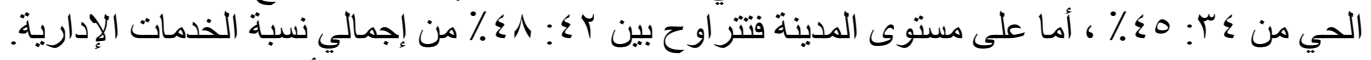

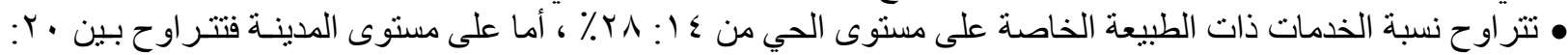

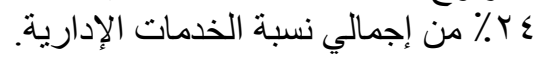

بعد أن تم رصد المعدلات التخطيطية للخدمات الإدارية في بعض الإول العربية والأجنبية، فيما يلي رسم بياني يقـارن بين المعدل

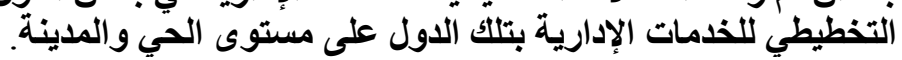

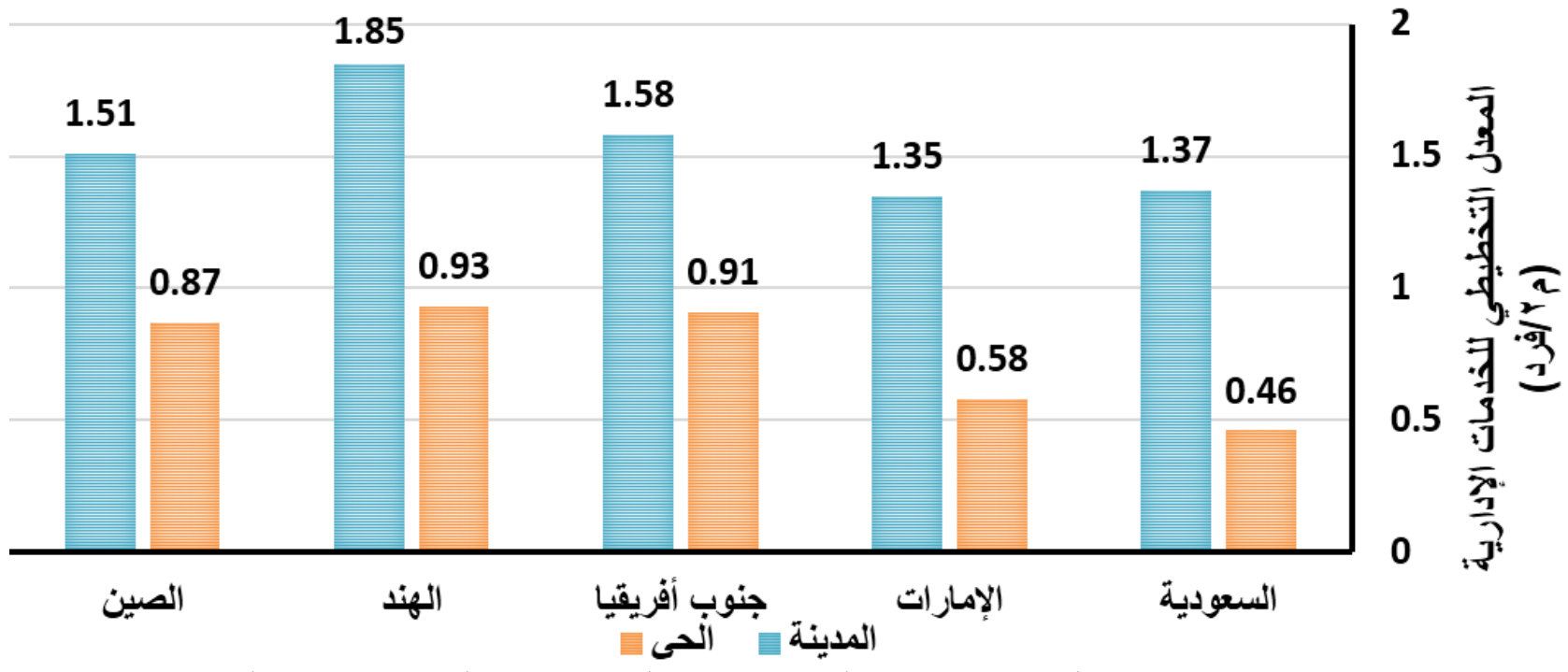

شكل (ء) ): مقارنة المعدلات التخطيطية للخدمات الإدارية بالدول المختلفة ـ المصدر: الباحثة

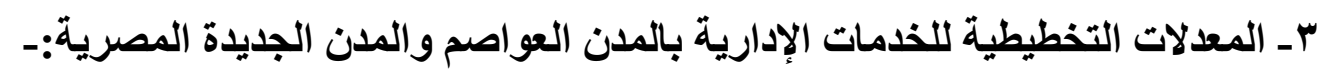

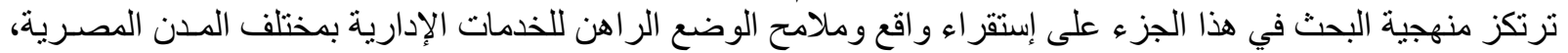

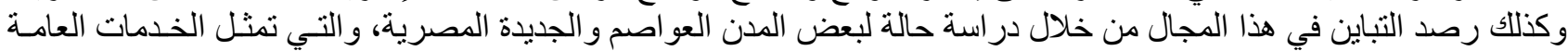

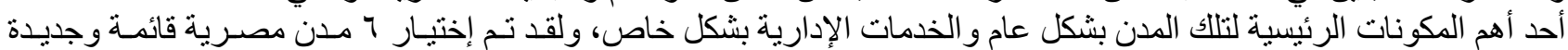

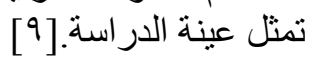

$$
\text { ب-1 أسباب إختيار مدن الدراسة: }
$$

لقد تم إختيار مدن الدار مدن الاسة وفقاً للمعايير الآتية:

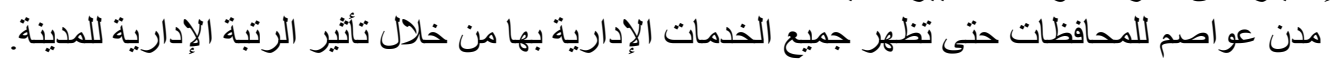

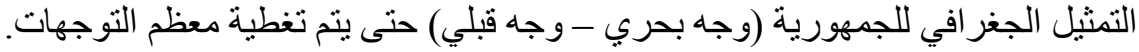

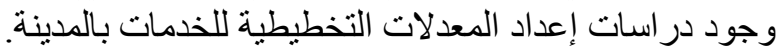
هر اسة الخدمات الإدارية بيعض المدن الجديدة في فترات زمنية بلة مختلفة (جيل أول - جيل ثاني - جيل ثالث). 


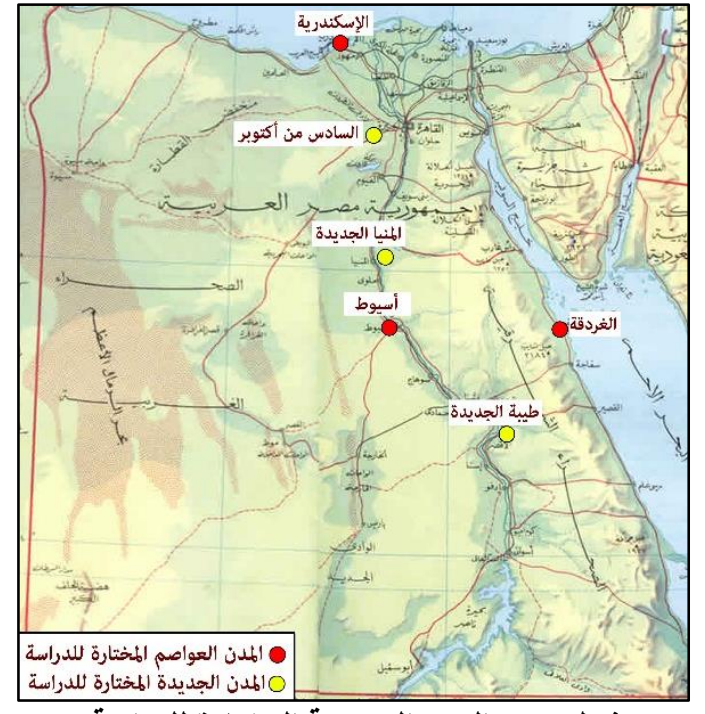

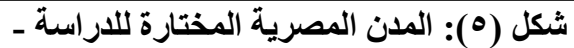
المصدر: الباحثة بتصرف عن الموقع الإلكتروني http://www.giza.gov.eg/country\% 20 map/default.aspx Access: 10/2/2020.

وتتمثل عينة الدر اسة في كل من:

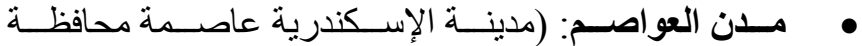

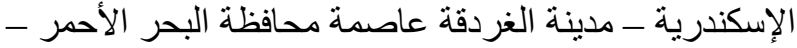
مدينة أسيوط عاصمة محافظة أسيوط).

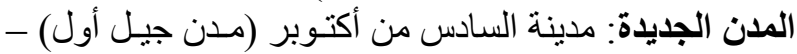

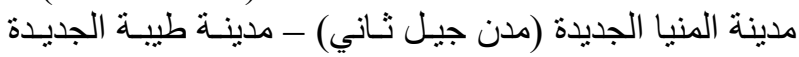

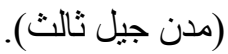

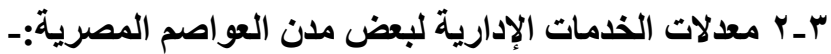

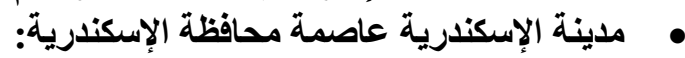

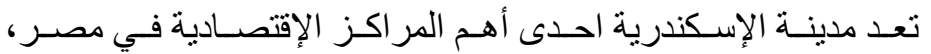

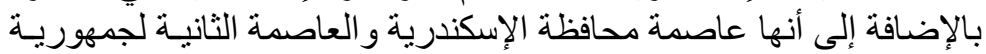

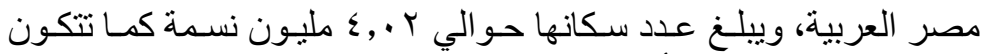

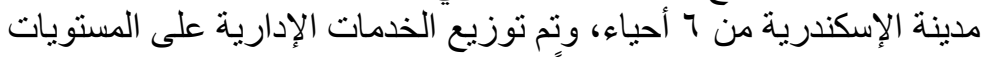

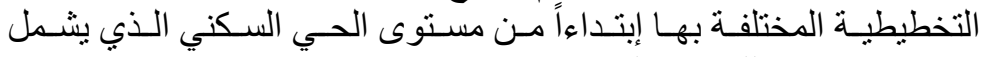

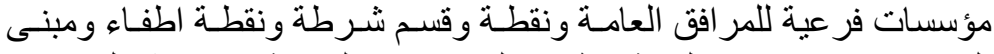

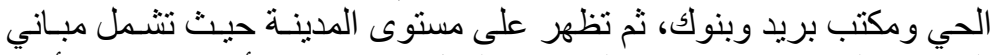

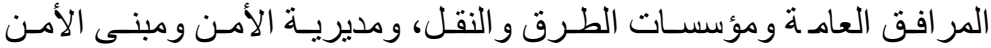

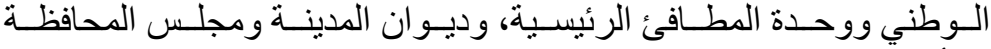
و الأحوآل المدنية ووحدة المرور الرئيسية ومركز البريد الرئيسي ومباني حكومية أخرى، بالإضـافة إلى المحساكم ومبنسى النيابـات وهيئة قضايا الدولة والبنك المركزي. [• (1)

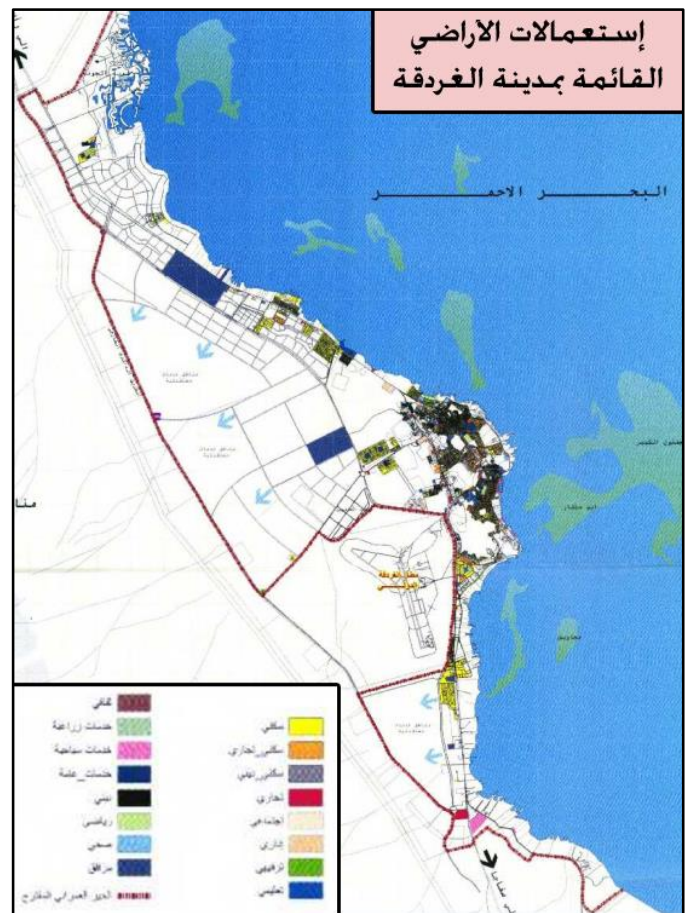

شكل (V): إستعمالات الأراضي القائمة بمدينة

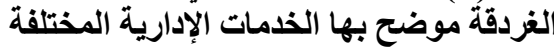

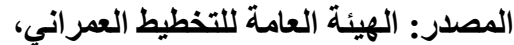

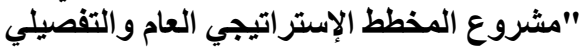

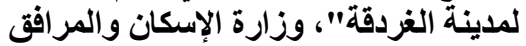

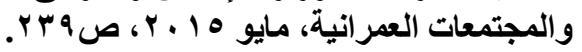

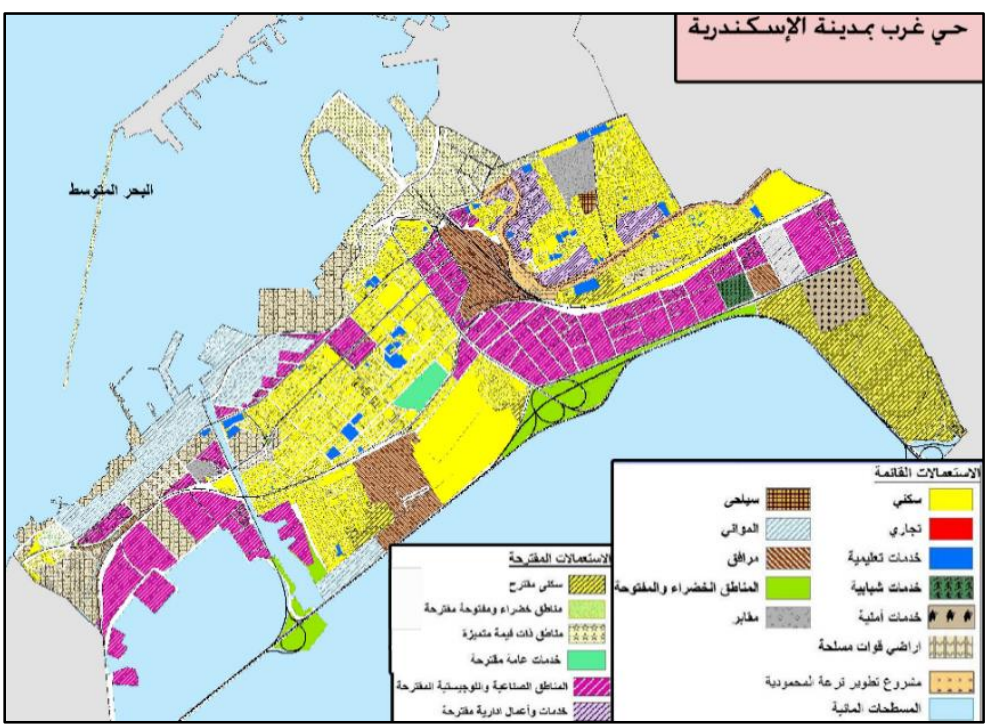

شكل (†)): إستعمالات الأراضي القائمة بحي غرب مدينة الإسكندرية موضح

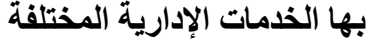

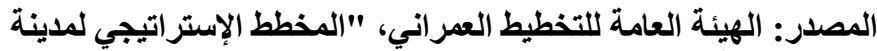

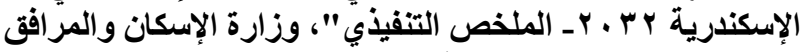

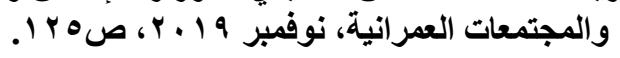

\section{مدينة الغردقة عاصمة محافظة البحر الأحمر:}

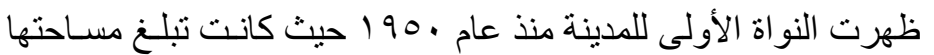

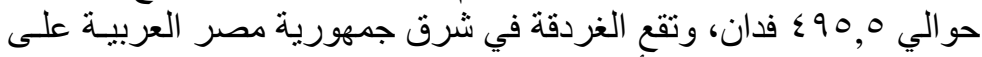

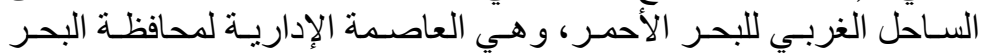

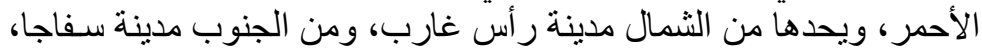

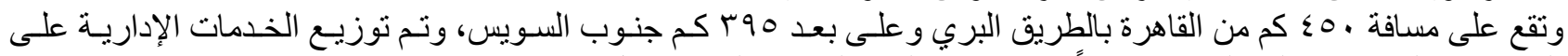

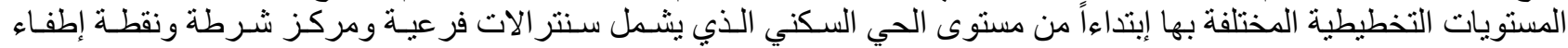
ومكتب بريد ومبنى الحي ومركز المعلومات و البنوك، ثم تظهر على مستوى المدينة من خـلال مؤسسـات المر افق العامـة ومديريـة 
الأمن و المطافئ ومجلس المدينة و المكاتب الحكوميـة المختلفـة والثـهر العقاري و الجواز ات ومكتب البريــ الرئيسـي و الجمـارك و المحاكم.[11)

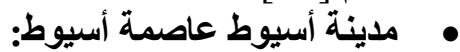

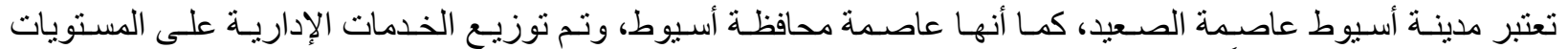

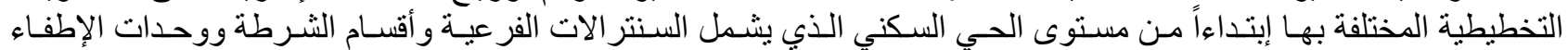

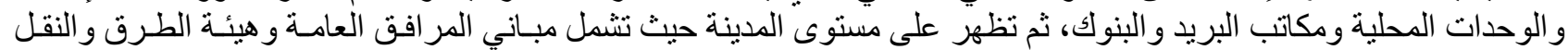

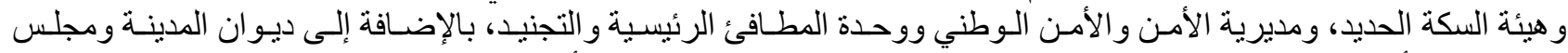

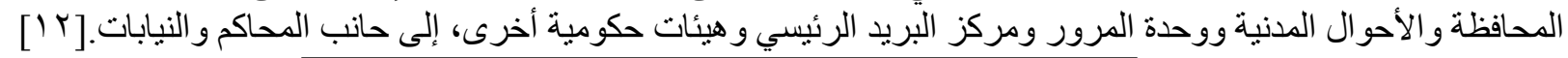

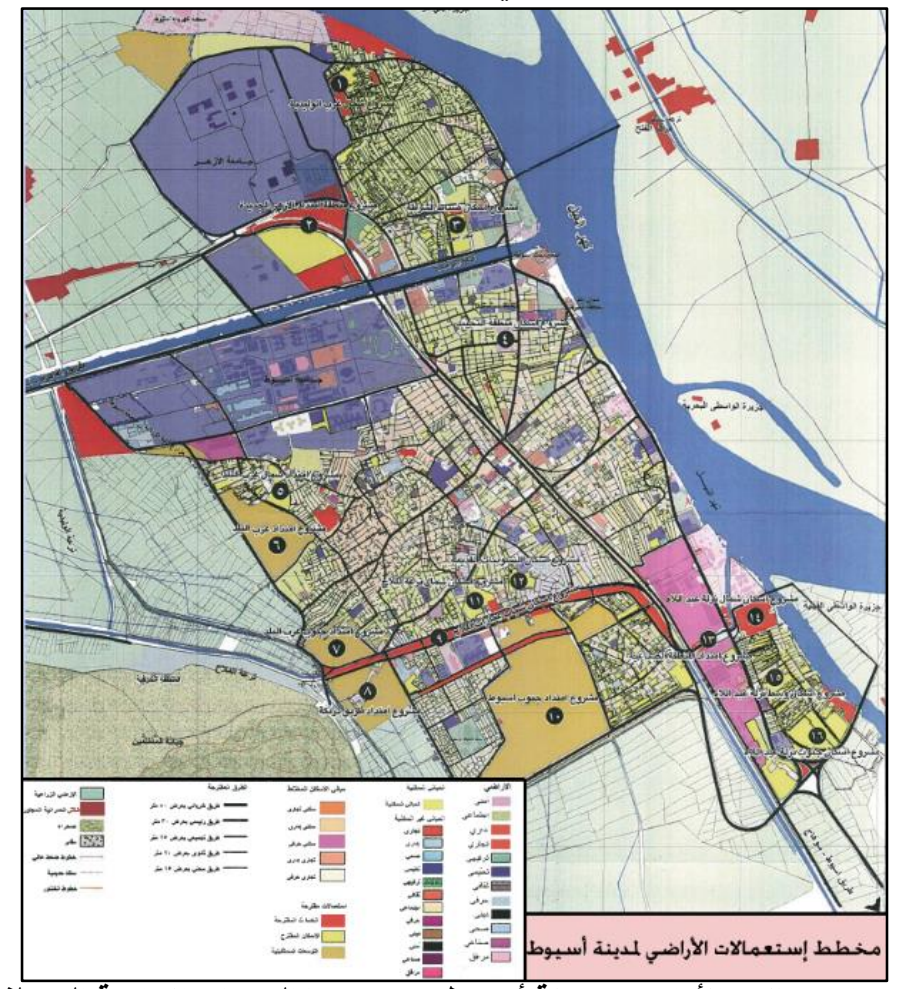

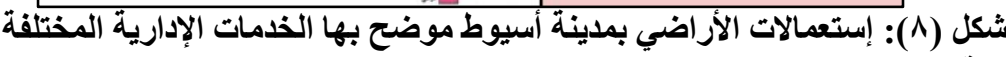

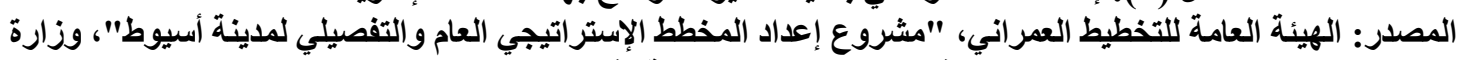

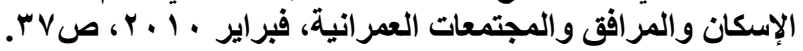

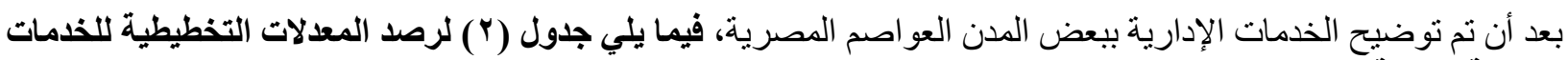

جدول (ץ): المعدلات التخطيطية للخدمات الإدارية بالمدن العواصم المصرية الإدارية القائمة بتلك المدن.

\begin{tabular}{|c|c|c|c|c|c|c|c|}
\hline \multicolumn{6}{|c|}{ المستوى التخطيطي } & \multirow[b]{3}{*}{ 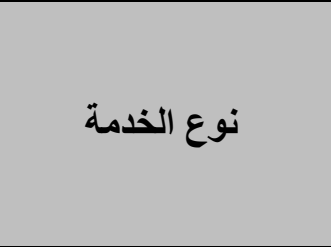 } & \multirow[b]{3}{*}{ مدن العواصم } \\
\hline \multicolumn{3}{|c|}{ المدينة } & \multicolumn{3}{|c|}{ الحي } & & \\
\hline أنسبة & نصيب الفرد من & $\begin{array}{l}\text { (المخدومين السكان } \\
\text { (ألف نسمة) }\end{array}$ & | النسبة & نصيب الفرد من & 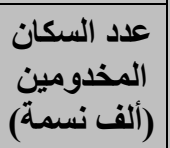 & & \\
\hline$\% 11$ & $\cdot, r$ & \multirow{4}{*}{$\leqslant 7 \cdot \leqslant$} & $\%$. & $\cdot, r_{1}$ & \multirow{4}{*}{$0 .-r$. } & خدمات مرافق عامة & \multirow{4}{*}{ الإسكندرية } \\
\hline$\%$ Y &., 0 & & $\%$ \% & $\cdot, \wedge$ & & خذمات السلامة العامة & \\
\hline$\%$ \% & $\cdot, 9$ & & $\% \varepsilon r$ & $1,1 \leqslant$ & & الخدمات الحكومية & \\
\hline$\% 17$ & $\cdot, r$ & & $\%$. & $\cdot$, or & & خدمات ذات طبيعة خاصة & \\
\hline \multicolumn{3}{|c|}{ على مستوى المدينة 9 , } & \multicolumn{3}{|c|}{ على مستوى الحي 9 ب, r } & \multicolumn{2}{|c|}{ إجمالي معدل الخدمات الإدارية } \\
\hline$\%$ \% & $\cdot, Y$ & \multirow{4}{*}{ IVT,q } & $\% 11$ & $\cdot, \cdot \wedge$ & \multirow{4}{*}{$r \Lambda$ - rV } & خدمات مرافق عامة & \multirow{4}{*}{ الغردقة } \\
\hline \%Yr & $\cdot, 1 Y$ & & $\% r v$ & .6 . YA & & خدمات السلامة العامة & \\
\hline$\%$ \% &., 19 & & $\%$ & $\cdot, r T$ & & الخدمات الحكومية & \\
\hline$\% \varepsilon$ & $\cdot, \cdot r$ & & $\% Y_{1}$ & $\cdot, 17$ & & خدمات ذات طبيعة خاصة & \\
\hline \multicolumn{3}{|c|}{ على مستوى المدينة ب ه, • } & \multicolumn{3}{|c|}{ على مستوى الحي •V, • } & \multicolumn{2}{|c|}{ إجمالي معدل الغدمات الإدارية } \\
\hline$\%$ Yo & $\cdot, \cdot 7$ & $0 . Y, \Sigma \wedge 9$ & $\% 9$ & $\cdot, \cdot 1$ & 00,1 & خدمات مرافق عامة & أسيوط \\
\hline
\end{tabular}


Prof. Noha Ahmed Nabil/ et al/ Engineering Research Journal 171 (September, 2021) AA 111 - AA 125

\begin{tabular}{|c|c|c|c|c|c|}
\hline$\% 10$ & $\cdot, \cdot \varepsilon$ & $\% \leqslant r$ & $\cdot, \Gamma q$ & خدمات السلامة العامة & \\
\hline$\%$ \% & $\cdot, 1$ & $\%$ & $\cdot, r q$ & الخدمات الحكومية & \\
\hline$\% 1 \wedge$ &., .0 & $\% 19$ & $\cdot, 1 \wedge$ & خدمات ذات طبيعة خاصة & \\
\hline \multicolumn{2}{|c|}{ على مستوى المدينة ه ب, . } & \multicolumn{2}{|c|}{ على مستوى الحي ؟ 9 ـ. } & \multicolumn{2}{|c|}{ إجمالي معدل الذامات الإدارية } \\
\hline \multicolumn{2}{|c|}{ على مستوى المدينة هץ, · _ 9, 1} & \multicolumn{2}{|c|}{ 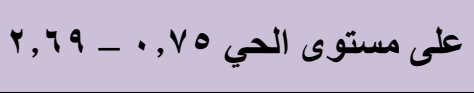 } & \multicolumn{2}{|c|}{ مستوسط معدل بالخدمات الإدارية على العولى } \\
\hline
\end{tabular}

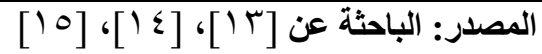

من خلال دراسة المعدلات التخطيطية للخدمات الإدارية ببعض المدن العواصم المصرية تم التوصل إلى الآتي:-

• تختلف المعدلات الواردة بين المدن المختلفة نتيجة تباين عمر المدينة وتـاريخ إنشـائها، بالإضـافة إلى مدى إكتمـال خدمات المختلفة بها خصوصاً في المدن الجديدة.

• تحقق مدينة الإسكندرية معدلات عالية من نصيب الفرد في الخدمات الإدارية نظر أ لأنها العاصمة المصرية الثانية إلى جانب أنها مدينة مليونية. • بقل نصيب الفرد من الخدمات الإدارية على مستوى المدينة عن نصيبه على مستوى الحي.

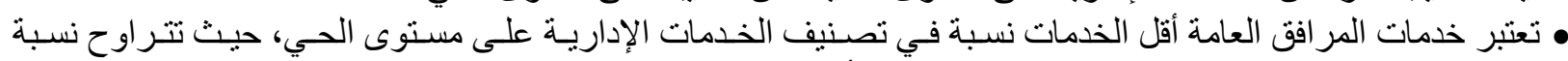

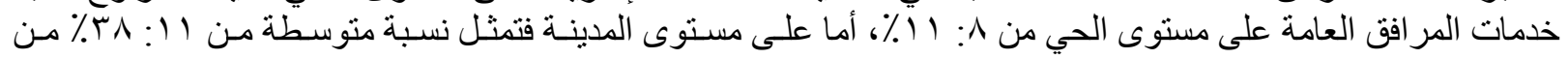
إجمالي نسبة الخدمات الإدارية.

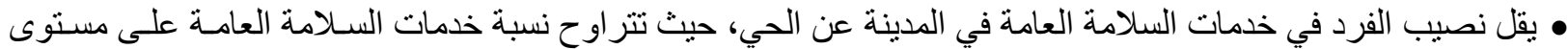

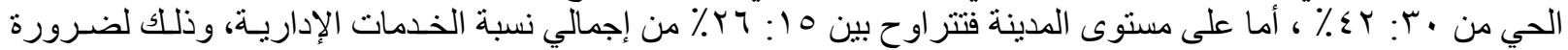

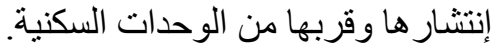

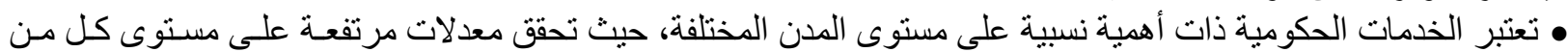

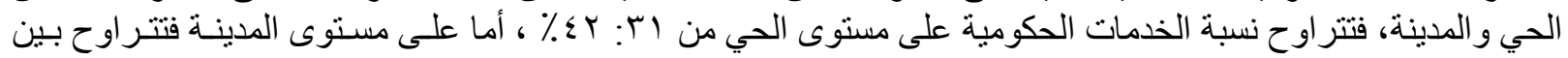

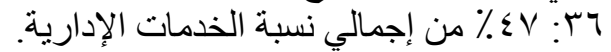

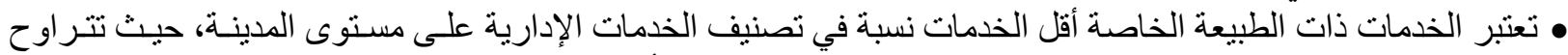

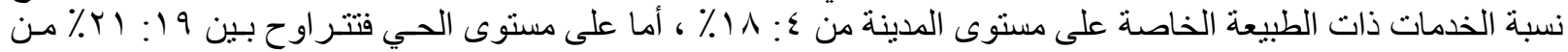
إجمالي نسبة الخدمات الإدارية.

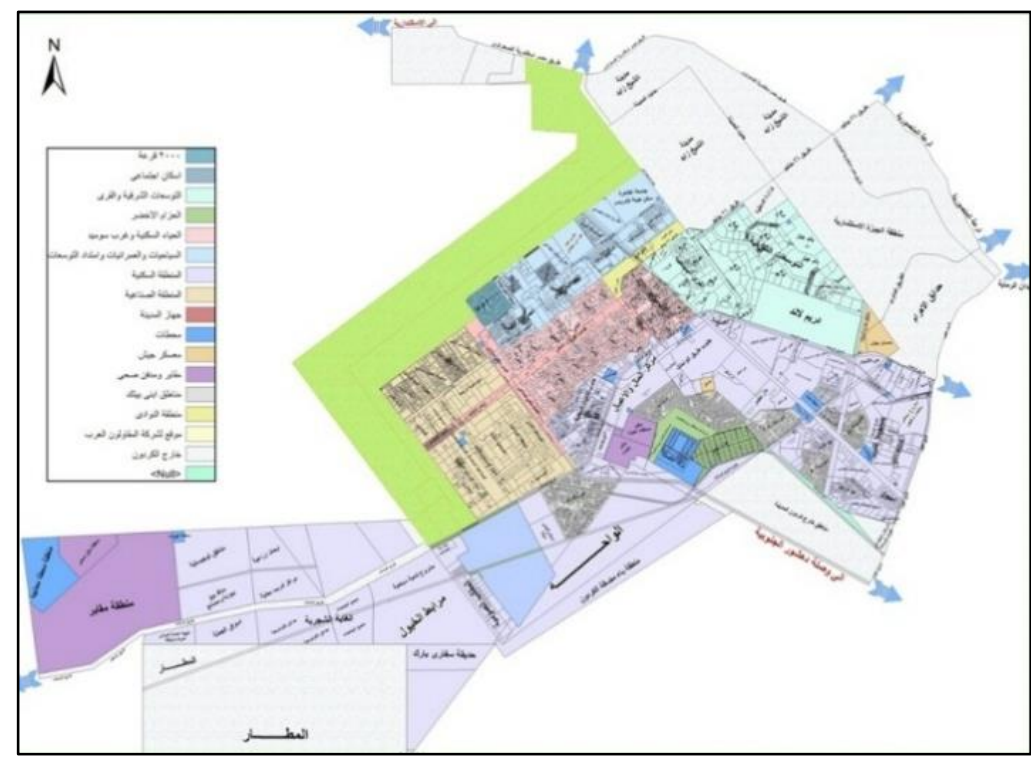

شكل (9): إستعمالات الأراضي بمدينة 9 أكتوبر موضح بها الخدمات الإدارية المختلفة

المصدر: http://www.6october.gov.eg/default.aspx Access:22/5/2021 at 1:00am.

r_M معدلات الخدمات الإدارية لبعض المدن الجديدة:• مدينة السادس من أكتوبر:

تعتبر مدينـه السـادس مـن أكتوبر مـن مـدن إنها

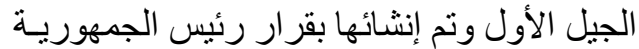

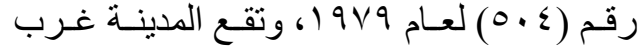

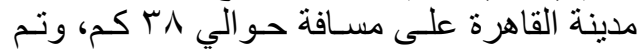

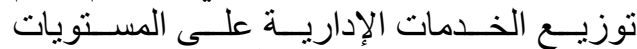
التخطيطية المختلفة بها إبتداءاً من مستوى الخدى الحي المي

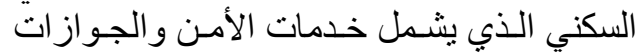

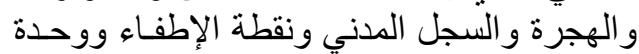

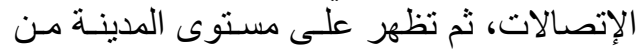

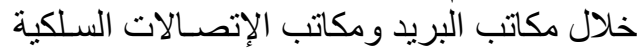

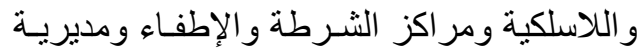

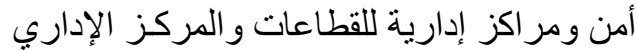

لإندينة. 


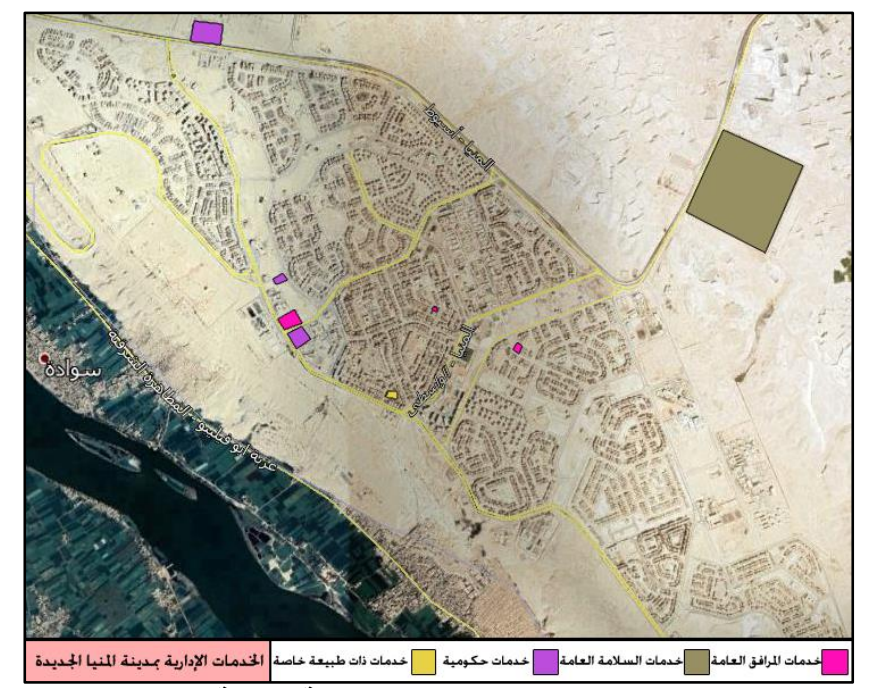

شكل ( • (1): الوضع الراهن للخدمات الإدارية بمدينة المنيا الجديدة

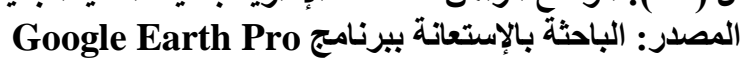

مدينة المنيا الجدياة:

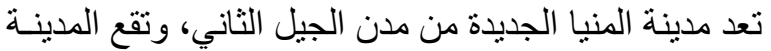

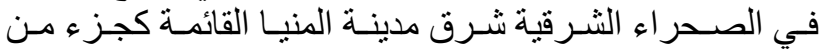

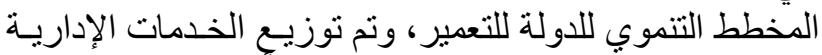

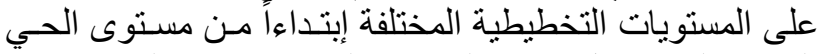

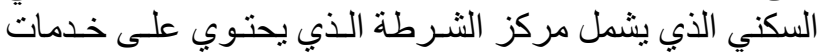

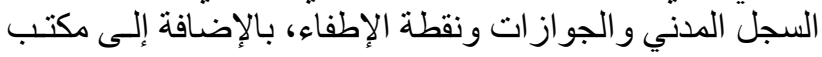

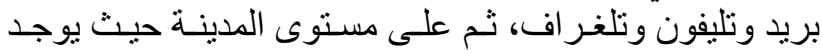
المركز الرئيسي للثرطة ومركز إطفاء ومركز البريد الرئيسي التيسي

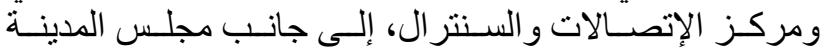

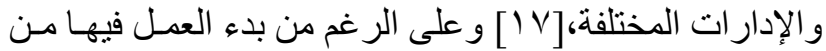

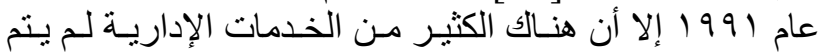

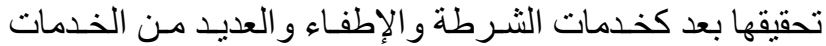

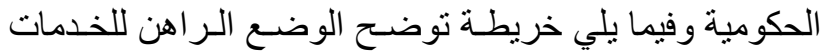
الإدارية بالمدينة.

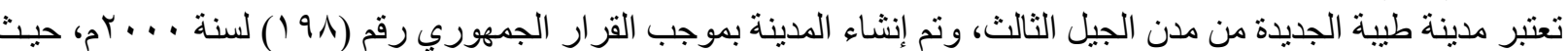

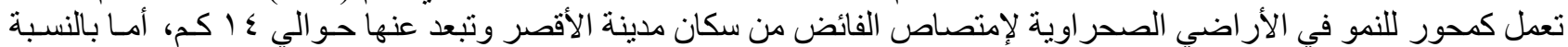

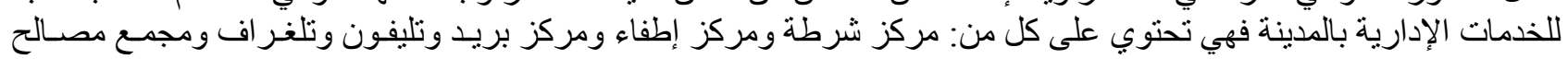
حكومي ومجلس المدينة. [11

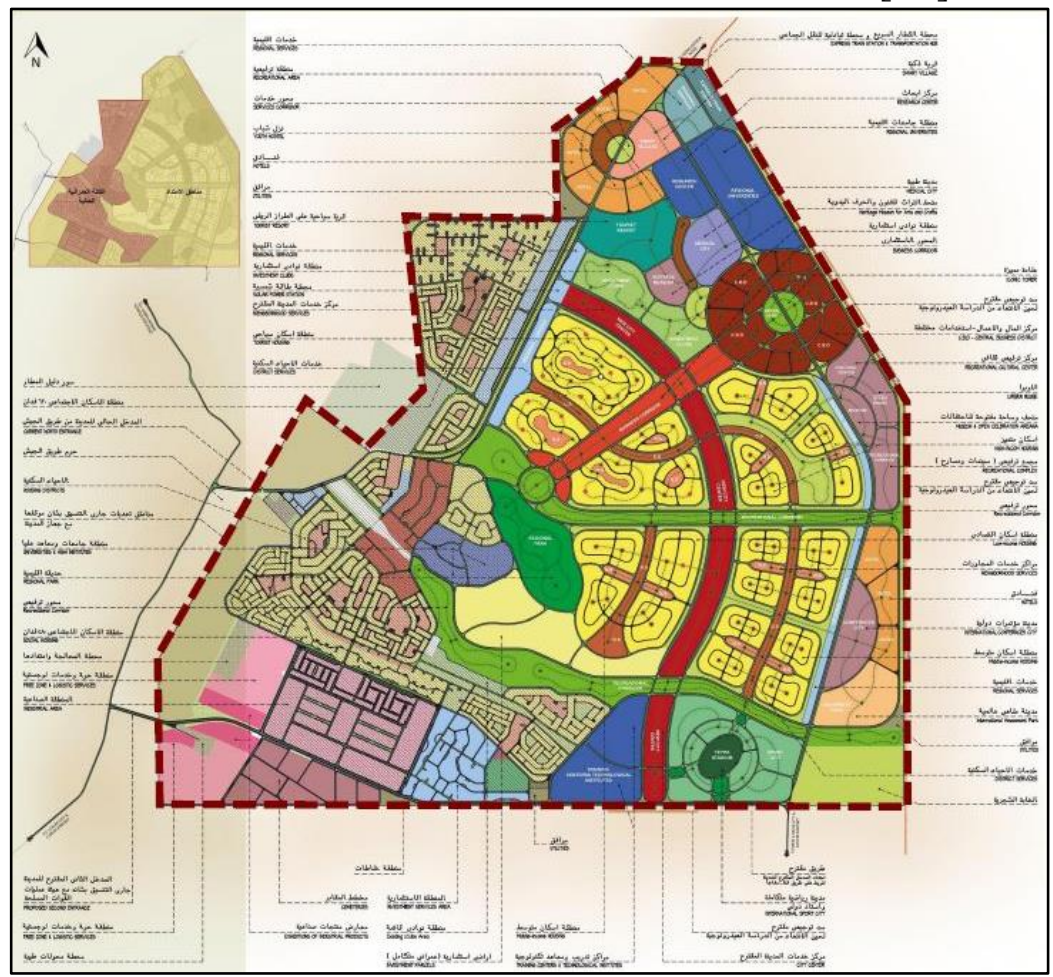

شكل (1 1) (1): المخطط العام لمدينة طيبة الجديدة موضح به الخدمات المختلفة ـ المصدر:

http://www.newcities.gov.eg/know_cities/tiba/default.aspx Access: 23/5/2021

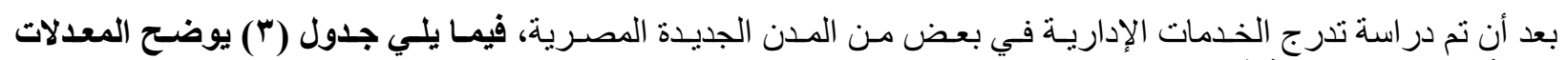
التخطيطة للخدمات الإدارية في تلكار المدن بشكل مفصل. 
Prof. Noha Ahmed Nabil/ et al/ Engineering Research Journal 171 (September, 2021) AA 111 - AA 125

جدول (r): المعدلات التخطيطية للخدمات الإدارية بالمدن الجديدة المصرية

\begin{tabular}{|c|c|c|c|c|c|c|c|}
\hline \multicolumn{6}{|c|}{ المستوى التخطيطي } & \multirow[b]{3}{*}{ نوع الخدمة } & \multirow[b]{3}{*}{ الجديدة } \\
\hline \multicolumn{3}{|c|}{ المدينة } & \multicolumn{3}{|c|}{ الحي } & & \\
\hline | النسبة & صيب الفرد مرة المساحة & | عدد السكان & | & نصيب الفرد من & 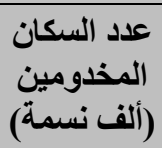 & & \\
\hline$\%$ \% & $\cdot, 11$ & \multirow{4}{*}{ ro. } & $\%$ \%r &., 01 & \multirow{4}{*}{ ro_ ro } & خدمات مرافق عامة & \multirow{4}{*}{ |أستادس | أكتوبر } \\
\hline$\%$ & $\cdot, \cdot r$ & & $\%$ & $1, Y 1$ & & خدمات السلامة العامة & \\
\hline$\% .01$ & $\cdot, r q$ & & $\% \vee$ & $\cdot, 17$ & & الخدمات الحكومية & \\
\hline$\%$ &., .0 & & $\% 1 \wedge$ & $\cdot, \varepsilon$ & & |خدمات ذات طبيعة خاصة & \\
\hline \multicolumn{3}{|c|}{ على مستوى المدينة ه, . } & \multicolumn{3}{|c|}{ على مستوى الحي r, rV } & \multicolumn{2}{|c|}{ إجمالي معدل الخدمات الإدارية } \\
\hline$\%$ \%. & $\cdot, \wedge 0$ & \multirow{4}{*}{ Ir. } & $\% 1 \leqslant$ & $\cdot, \mathrm{VO}$ & \multirow{4}{*}{$\varepsilon \cdot-\Gamma$} & خدمات مرافق عامة & \multirow{4}{*}{ الجديدة } \\
\hline$\% 1 \leq$ & $\cdot, \varepsilon$ & & $\% \vee Y$ & $\cdot, \varepsilon$ & & خدمات السلامة العامة & \\
\hline$\%$ or & $1, \leqslant 0$ & & $\% 1 \leq$ & $\cdot, \mathrm{VO}$ & & الخدمات الحكومية & \\
\hline$\%$ ๘ & $\cdot, 1$ & & - & لا يوجد & & خدمات ذات طبيعة خاصة & \\
\hline \multicolumn{3}{|c|}{ على مستوى المدينة r, ب } & \multicolumn{3}{|c|}{ على مستوى الحي 9,1} & \multicolumn{2}{|c|}{ إجمالي معدل الخدمات الإدارية } \\
\hline$\%$ & $\cdot, 1$ & \multirow{4}{*}{$\varepsilon}$. & - & لا يوجد & & خدمات مرافق عامة & \multirow{4}{*}{ الجذيدة } \\
\hline$\%$ \%q & $\cdot, 1 \leq$ & & - & لا يوجد & & خدمات السلامة العامة & \\
\hline$\% 01$ & $\cdot, r_{0}$ & & - & لا يوجد & & الخدمات الحكومية & \\
\hline- & لا يوجد & & - & لا لا يوجد & & خدمات ذات طبيعة خاصة & \\
\hline \multicolumn{3}{|c|}{ على مستوى المدينة 9 ؟ , . } & \multicolumn{3}{|c|}{ لا يوجد } & \multicolumn{2}{|c|}{ إجمالي معدل الخدمات الإدارية } \\
\hline \multicolumn{3}{|c|}{ 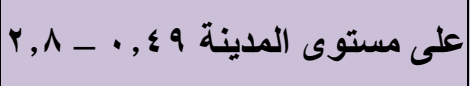 } & \multicolumn{3}{|c|}{ 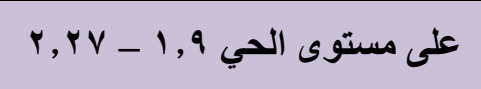 } & \multicolumn{2}{|c|}{ متوسط معدل بالخدمات الإدارية على المدية } \\
\hline
\end{tabular}

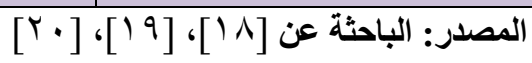

من خلال دراسة المعدلات التخطيطية للخدمات الإدارية ببعض المدن الجديدة المصرية تم التوصل إلى الآتي:-

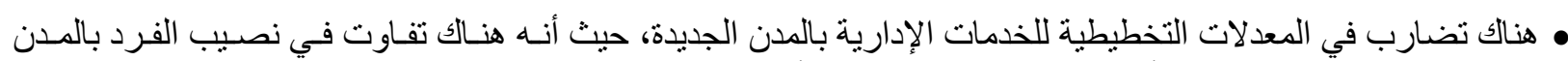

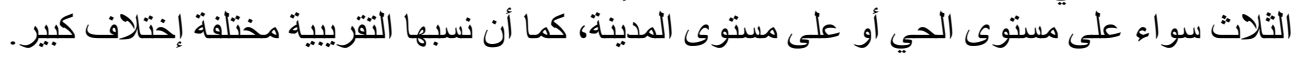

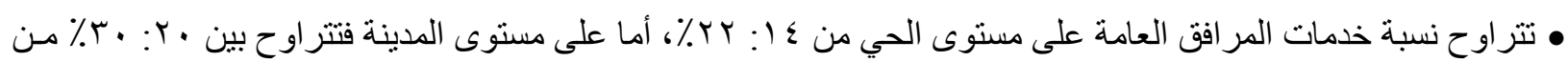
إجمالي نسبة الخدمات الإدارية.

• تحقق الخدمات العامة النسبة الأكبر من الخدمات الإدارية على مستوى الحي لإهتمام المدن الجديدة بتوفير عامل الأمن، كمـا

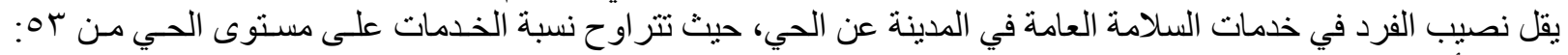

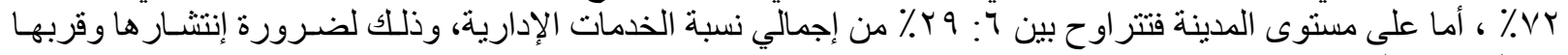
من الوحدات السكنية.

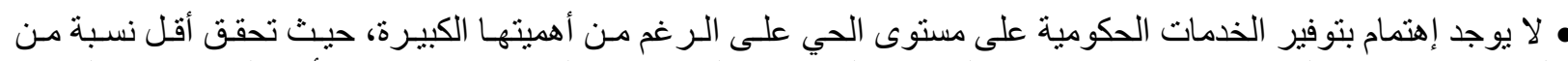

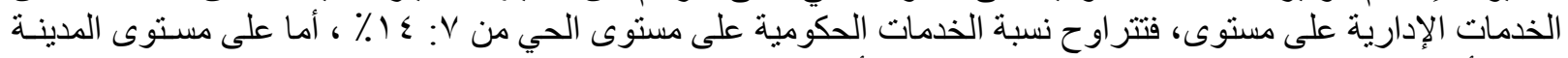

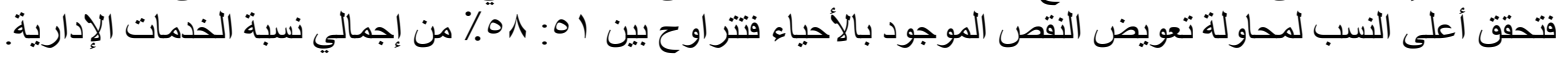

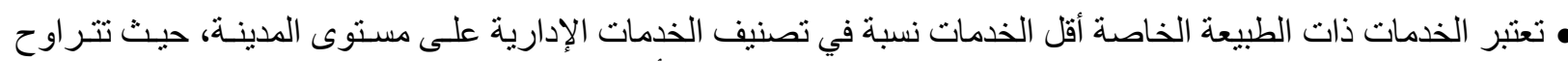

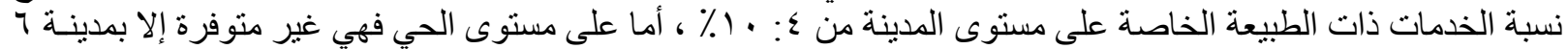

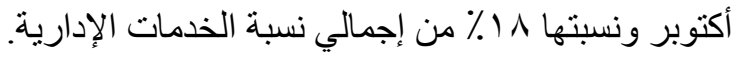




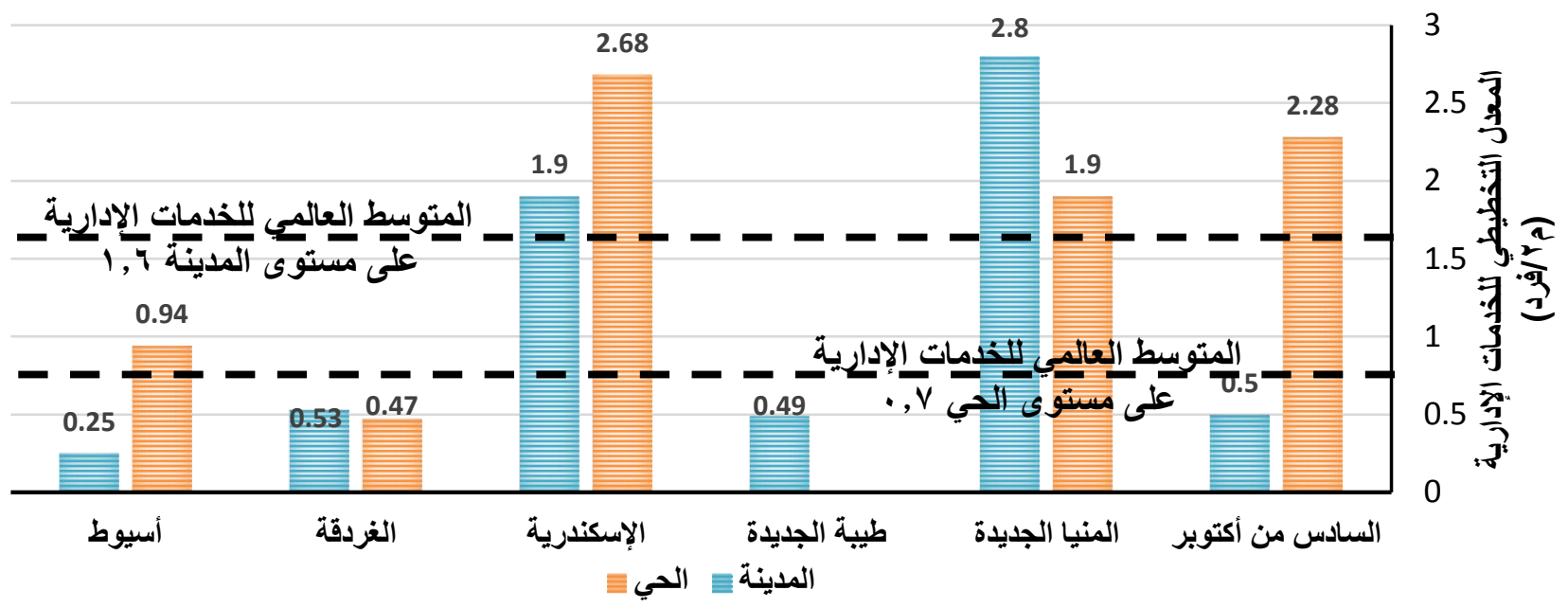

شكل (r ا ): مقارنة بين المعدلات التخطيطية لإجمالي الخدمات الإدارية بمدن الدراسة ـ المصدر: الباحثة

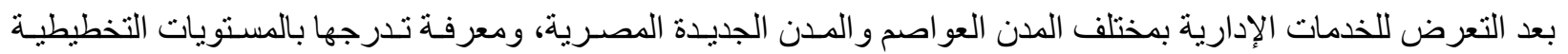

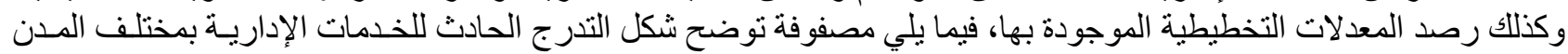

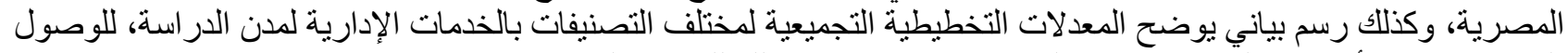

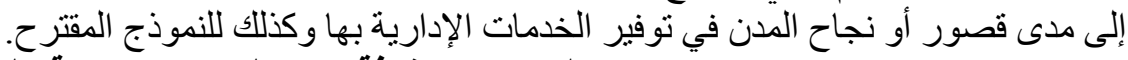

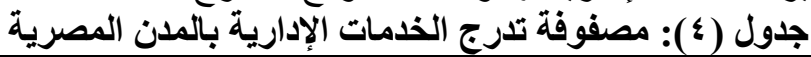

\begin{tabular}{|c|c|c|c|c|c|c|c|c|c|c|c|c|c|c|c|c|c|c|c|c|c|c|c|}
\hline \multicolumn{5}{|c|}{ خدمات ذات طبيعة خاصة } & \multicolumn{8}{|c|}{ خذمات حكومية } & \multicolumn{4}{|c|}{ خدمات السلامة العامة } & \multicolumn{4}{|c|}{ خدمات المرافق العامة } & \multirow[b]{2}{*}{ التخطيطي |ل } & \multirow{2}{*}{\multicolumn{2}{|c|}{ المدن المصرية }} \\
\hline $\begin{array}{l}\text { 年 } \\
\frac{1}{4} \\
\overline{3} \\
\overline{3}\end{array}$ & नें & 高 & 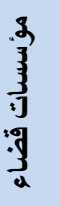 & 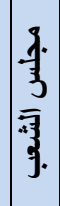 & $\frac{\hat{d}}{\sqrt[3]{3}}$ & 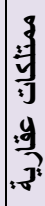 & 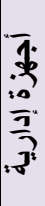 & $\frac{5}{3}$ & 亲: & $\begin{array}{l}3 \\
3 \\
\vdots \\
\vdots \\
\frac{3}{3} \\
\frac{3}{5}\end{array}$ & $\frac{7}{3}$ & 寻 & 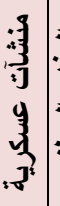 & 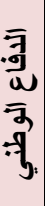 & $\begin{array}{l}\cdot \frac{7}{3} \\
\overline{3} \\
\overline{3} \\
3\end{array}$ & 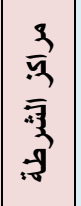 & 承 & 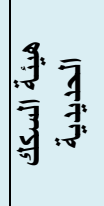 & 象高 & 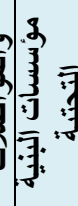 & & & \\
\hline & $\sqrt{ }$ & & & & & $\sqrt{ }$ & $\sqrt{ }$ & & $\sqrt{ }$ & $\sqrt{ }$ & & $\sqrt{ }$ & & & $\sqrt{ }$ & $\sqrt{ }$ & & & & $\sqrt{ }$ & حي - - - حي & & \\
\hline & $\sqrt{ }$ & & $\sqrt{ }$ & & $\sqrt{ }$ & $\sqrt{ }$ & $\sqrt{ }$ & $\sqrt{ }$ & $\sqrt{ }$ & $\sqrt{ }$ & $\sqrt{ }$ & & $\sqrt{1}$ & $\sqrt{ }$ & $\sqrt{ }$ & $\sqrt{ }$ & $\sqrt{ }$ & $\sqrt{ }$ & $\sqrt{ }$ & $\sqrt{ }$ & مدينة & & \\
\hline & $\sqrt{ }$ & & & & & & $\sqrt{ }$ & & $\sqrt{ }$ & & & $\sqrt{ }$ & & & & $\sqrt{ }$ & & & & $\sqrt{ }$ & حي & & \\
\hline & & & $\sqrt{ }$ & & $\sqrt{ }$ & $\sqrt{ }$ & $\sqrt{ }$ & $\sqrt{ }$ & $\sqrt{ }$ & $\sqrt{ }$ & $\sqrt{ }$ & & $\sqrt{ }$ & $\sqrt{ }$ & $\sqrt{ }$ & $\sqrt{ }$ & $\sqrt{ }$ & & $\sqrt{ }$ & $\sqrt{ }$ & مدينة & & \\
\hline & $\sqrt{ }$ & & & & & & $\sqrt{ }$ & & $\sqrt{ }$ & & & $\sqrt{ }$ & & & $\sqrt{ }$ & $\sqrt{ }$ & & & & $\sqrt{ }$ & حي & & \\
\hline & $\sqrt{ }$ & & $\sqrt{ }$ & & & $\sqrt{ }$ & $\sqrt{ }$ & & $\sqrt{ }$ & $\sqrt{ }$ & $\sqrt{ }$ & & $\sqrt{ }$ & $\sqrt{ }$ & $\sqrt{ }$ & $\sqrt{ }$ & $\sqrt{ }$ & $\sqrt{ }$ & $\sqrt{ }$ & $\sqrt{ }$ & مدينَة & & \\
\hline & $\sqrt{ }$ & & & & & & & & $\sqrt{ }$ & & & $\sqrt{ }$ & & & $\sqrt{ }$ & $\sqrt{ }$ & & & & $\sqrt{ }$ & حي & & \\
\hline & $\sqrt{ }$ & & $\sqrt{ }$ & & & $\sqrt{ }$ & $\sqrt{ }$ & & $\sqrt{ }$ & $\sqrt{ }$ & $\sqrt{ }$ & & & & $\sqrt{ }$ & $\sqrt{ }$ & $\sqrt{ }$ & & $\sqrt{ }$ & $\sqrt{ }$ & مدينة & & $\overline{7}$ \\
\hline & & & & & & & & & $\sqrt{ }$ & $\sqrt{ }$ & & $\sqrt{ }$ & & & & & & & & $\sqrt{ }$ & حي & & \\
\hline & $\sqrt{ }$ & & $\sqrt{ }$ & & & & $\sqrt{ }$ & & $\sqrt{ }$ & $\sqrt{ }$ & $\sqrt{ }$ & & $\sqrt{ }$ & & & $\sqrt{ }$ & & & & $\sqrt{ }$ & مدينة & a) & \\
\hline & & & & & & & & & & & & & & & & & & & & & حي & ط طبة II & \\
\hline & & & & & & & $\sqrt{ }$ & & $\sqrt{ }$ & $\sqrt{ }$ & $\sqrt{ }$ & & & & $\sqrt{ }$ & $\sqrt{ }$ & & & & $\sqrt{ }$ & مدينة & & \\
\hline
\end{tabular}

المصدر: الباحثة

من خلال الجدول السابق الموضح لتدرج الخدمات الإدارية ومقارنة المعدلات التخطيطية ببعض المدن العواصـم و الجديدة بمصر تم التوصل إلى الآتي:-

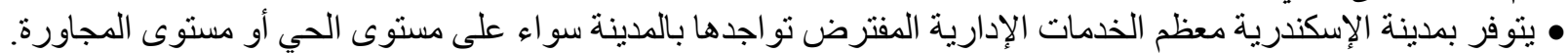

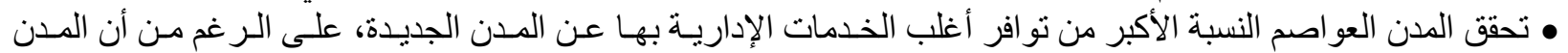

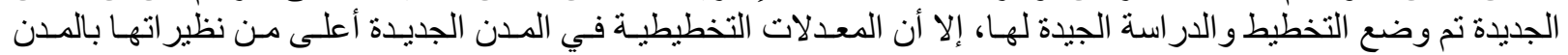

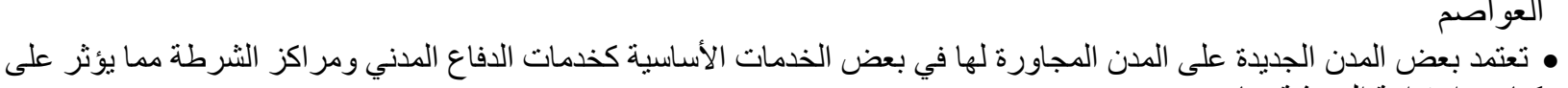
كفاءة و إستدامة المعيشة بها.

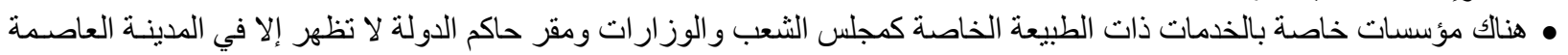
للادولة (القاهرة). • هناك قصور في توفير الخدمات الإدارية بالمدن العو اصم على مستوى الحي عن توافر ها بالمدن الجديدة. • صغر حجم السكان بالنسبة للمستوى التخطيطي قد لا يسمح بتوفير الخدمات المختلفة وخصوصاً الخدمات الإقليمية. 


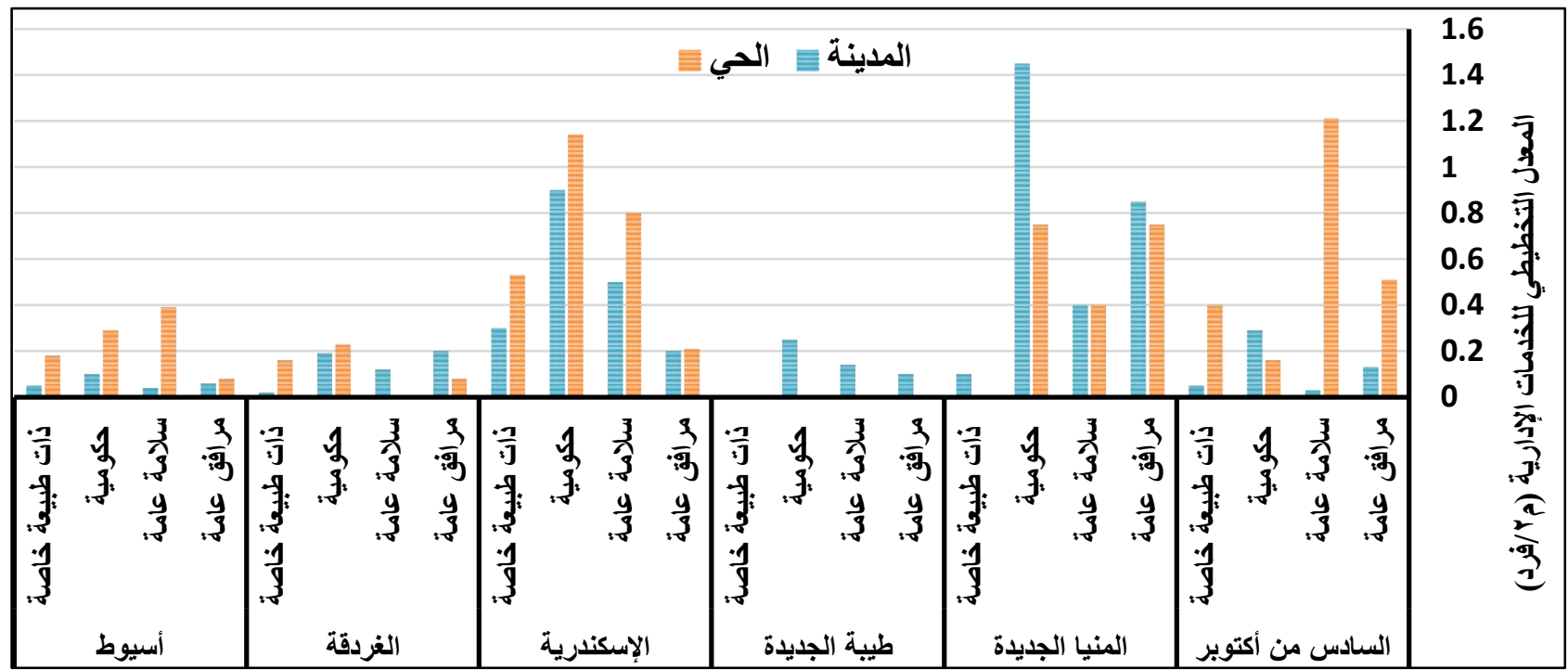

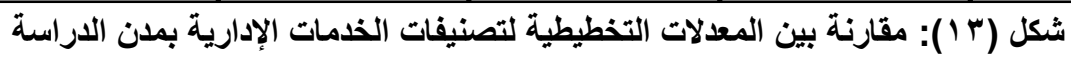
المصدر: الباحثة

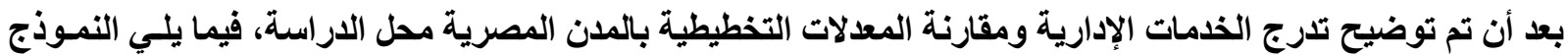

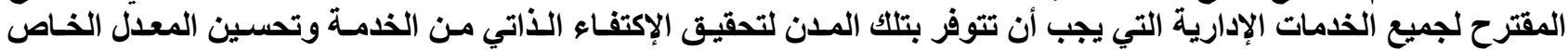

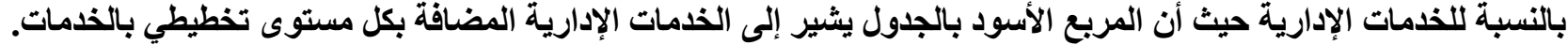

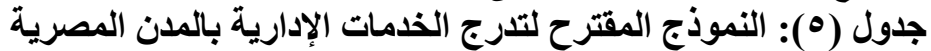

\begin{tabular}{|c|c|c|c|c|c|c|c|c|c|c|c|c|c|c|c|c|c|c|c|c|c|}
\hline \multicolumn{5}{|c|}{ |خدمات ذات طبيعة خاصة } & \multicolumn{7}{|c|}{ خدمات حكومية } & \multicolumn{3}{|c|}{ |خدمات السلامة العامة } & \multicolumn{4}{|c|}{ خدمات المرافق العامة } & \multirow[b]{2}{*}{ |التخطيطوى } & \multirow{2}{*}{\multicolumn{2}{|c|}{ المدن المصرية }} \\
\hline 年 & नें & $\stackrel{\frac{3}{3}}{\frac{3}{3}}$ & \$ & $\begin{array}{l}\frac{3}{3} \\
\frac{3}{3} \\
\beta .\end{array}$ & 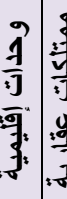 & 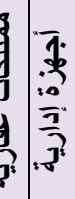 & 方 & 丞 & $\begin{array}{c}\frac{3}{3} \\
3 \\
\frac{2}{3} \\
\frac{3}{3} \\
\bar{j}\end{array}$ & $\begin{array}{l}\frac{7}{3} \\
\overline{3} \\
\overline{7} \\
\underbrace{*}\end{array}$ & 蛹 & 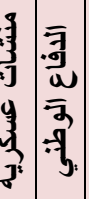 & 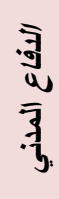 & 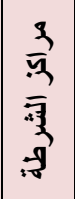 & 承 & 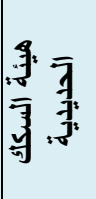 & 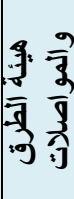 & 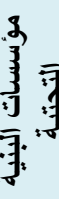 & & & \\
\hline & $\sqrt{ }$ & & & & 1 & $\sqrt{ } \sqrt{ }$ & & $\sqrt{ }$ & $\sqrt{ }$ & & $\sqrt{ }$ & & $\sqrt{ }$ & $\sqrt{ }$ & & & & $\sqrt{ }$ & حي & \multirow{6}{*}{\multicolumn{2}{|c|}{ لَ }} \\
\hline & $\sqrt{ }$ & & $\sqrt{ }$ & & $\sqrt{ }$ & $\sqrt{ } \sqrt{ }$ & $\sqrt{ }$ & $\sqrt{ }$ & $\sqrt{ }$ & $\sqrt{ }$ & & $\sqrt{ } \sqrt{ } \mid$ & $\sqrt{ }$ & $\sqrt{ }$ & $\sqrt{ }$ & $\sqrt{ }$ & $\sqrt{ }$ & $\sqrt{ }$ & مدينة & & \\
\hline & $\sqrt{1}$ & & & & & $\sqrt{ }$ & & $\sqrt{ }$ & & & $\sqrt{ }$ & & & $\sqrt{ }$ & & & & $\sqrt{ }$ & حي & & \\
\hline & & & $\sqrt{ }$ & & $\sqrt{ }$ & $\sqrt{ } \sqrt{ }$ & $\sqrt{ }$ & $\sqrt{ }$ & $\sqrt{ }$ & $\sqrt{ }$ & & $\sqrt{ } \sqrt{ } \mid$ & $\sqrt{ }$ & $\sqrt{ }$ & $\sqrt{ }$ & & $\sqrt{ }$ & $\sqrt{ }$ & مدينَة & & \\
\hline & $\sqrt{ }$ & & & & & & & $\sqrt{ }$ & & & $\sqrt{ }$ & & $\sqrt{ }$ & $\sqrt{ }$ & & & & $\sqrt{ }$ & حي & & \\
\hline & $\sqrt{ }$ & & $\sqrt{ }$ & & & $\sqrt{ }$ & & $\sqrt{ }$ & $\sqrt{ }$ & $\sqrt{ }$ & & \begin{tabular}{l|l|}
$\sqrt{ }$ & $\sqrt{ }$
\end{tabular} & $\sqrt{ }$ & $\sqrt{ }$ & $\sqrt{ }$ & $\sqrt{ }$ & $\sqrt{ }$ & $\sqrt{ }$ & مدينة & & \\
\hline & $\sqrt{ }$ & & & & & & & $\sqrt{ }$ & 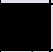 & & $\sqrt{ }$ & & $\sqrt{ }$ & $\sqrt{ }$ & & & & $\sqrt{ }$ & حي - - حي & \multirow{2}{*}{ צ أكتوبر } & \multirow{5}{*}{ 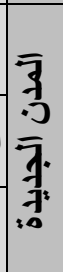 } \\
\hline & $\sqrt{ }$ & & $\sqrt{ }$ & & & $\sqrt{ }$ & & $\sqrt{ }$ & $\sqrt{ }$ & $\sqrt{ }$ & & & $\sqrt{ }$ & $\sqrt{ }$ & $\sqrt{ }$ & & $\sqrt{ }$ & $\sqrt{ }$ & مدينّة & & \\
\hline & & & & & & & & $\sqrt{ }$ & $\sqrt{1}$ & & $\sqrt{ }$ & & & & & & & $\sqrt{ }$ & حى & \multirow{2}{*}{ |المنيا الجديدة } & \\
\hline & $\sqrt{ }$ & & $\sqrt{ }$ & & & & & $\sqrt{ }$ & $\sqrt{ }$ & $\sqrt{ }$ & & $\sqrt{ }$ & & $\sqrt{ }$ & & & & $\sqrt{ }$ & مدينَة & & \\
\hline & & & & & & & & 1 & & & & & & & & & & & حي & طيبة الجديدة - & \\
\hline & & & & & & & & V & & V & & & V & $\frac{V}{-0}$ & & & & $v$ & مديك" & & \\
\hline
\end{tabular}

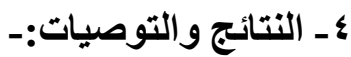

من خلال الدر اسة النظرية وتحليل عدد مـن المـدن العواصـم المصـرية مـن خـلال مقارنـة المعدلات التخطيطيـة للخدمات

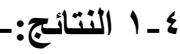
الإدارية بكل منهم، تم التوصل النوالن إلى الآتي:-

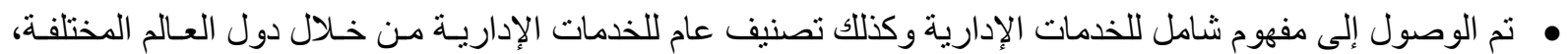

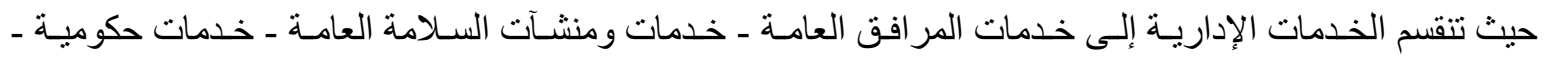


تم التوصل إلى متوسط عالمي تقريبي للخدمات الإدارية من خلال دراسة المدن العالمية المختلفة، حيث أن نصيب الفرد

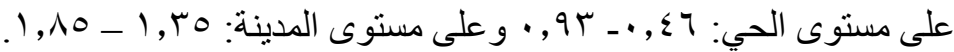

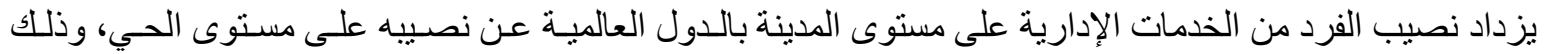

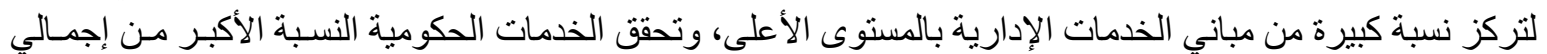

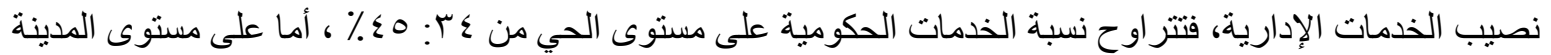

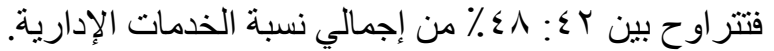

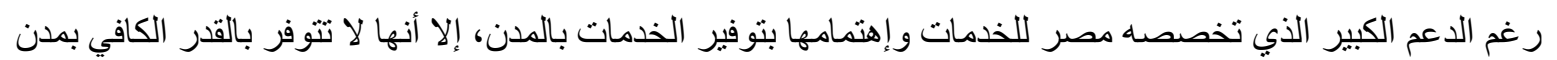

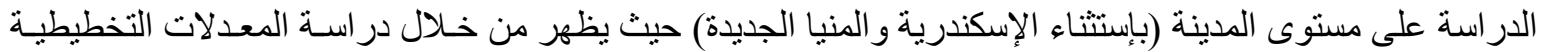

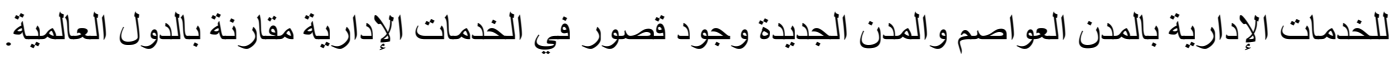

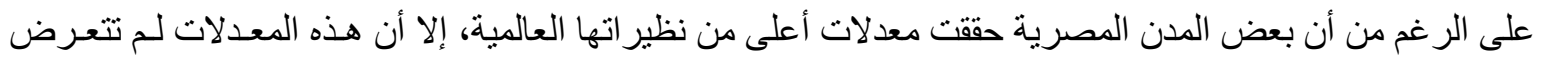

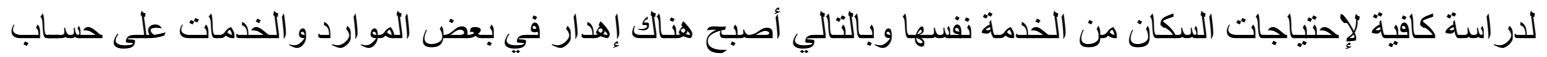

خدمات أخرى هنالك قصور فيها.

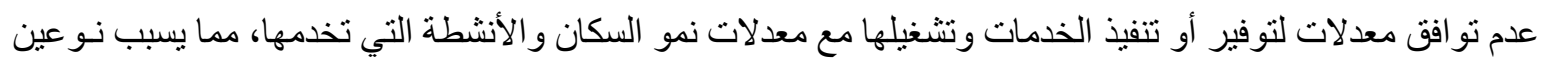

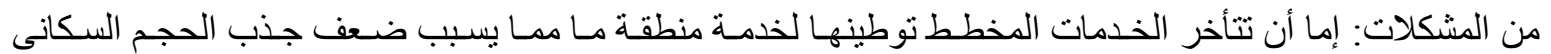

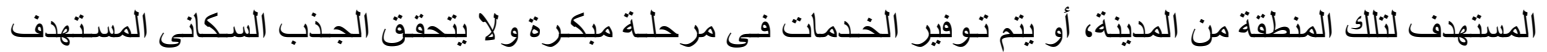

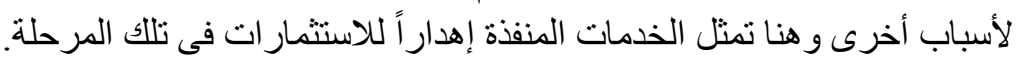

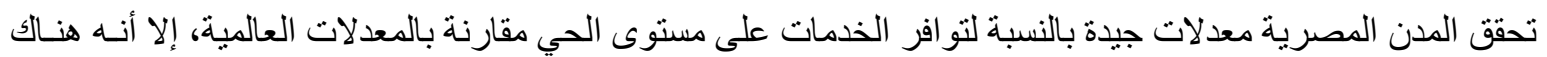

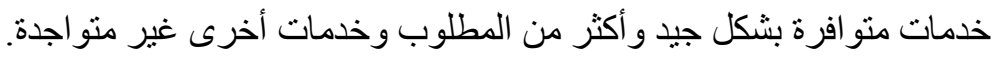

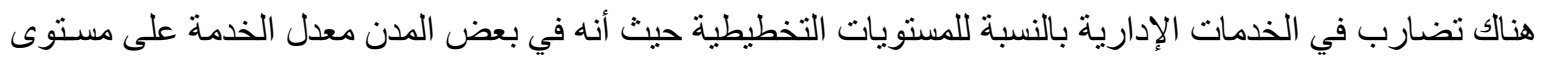

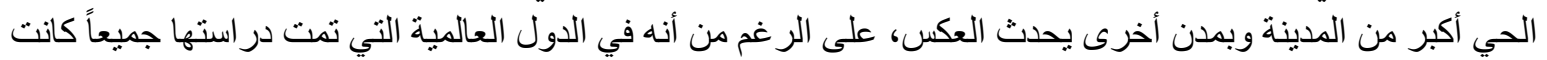

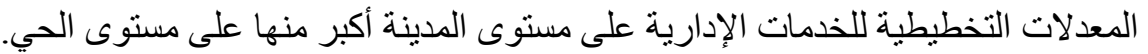

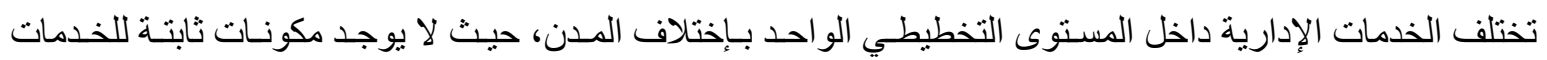
الإدارية سواء الجديدة أو القائمة. هناك تفاوت كبير في عدد السكان سو اء على المستوى التخطيطي نفسه أو بين المستويات التخطيطية.

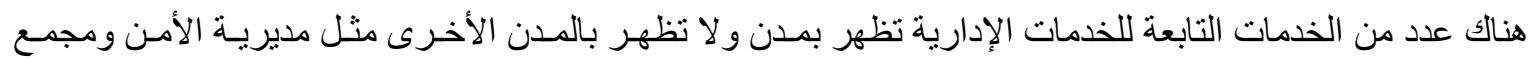

• در اسة المتغيرات المختلفة المؤثرة على المعدلات التخطيطية للخدمات الإدارية ووضعها في الإعنبار قبل تصميمها في المدن الجديدة. رصد الوضع الر اهن للخدمات الإدارية بالمدن القائمة و المشاكل المتعلقة بهاووضع الخطط اللازمة لتطوير ها أو زيـادة

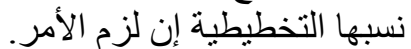
ز زيادة الإهتمام من قبل الدولة بتحديث المعدلات التخطيطية للخدمات الإدارية و إختيار ها على أسس ومحددات و اضحة.

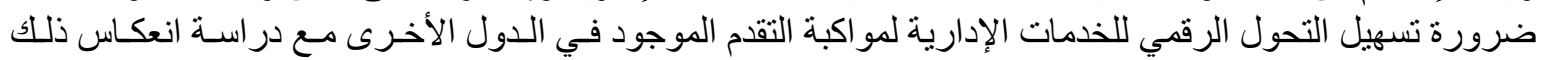
على المعدلات التخطيطية للخدمات الإندارية. • الإستفادة من تجارب الدول المتقدمة في مجال تقديم الخدمات الإدارية مع در اسة إمكانية التطبيق في مصر.

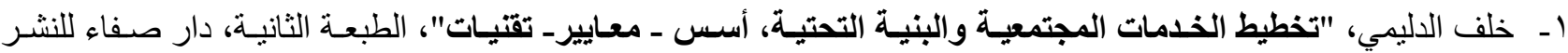

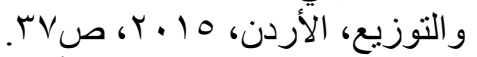

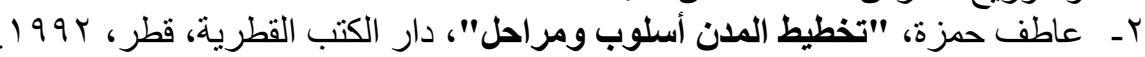

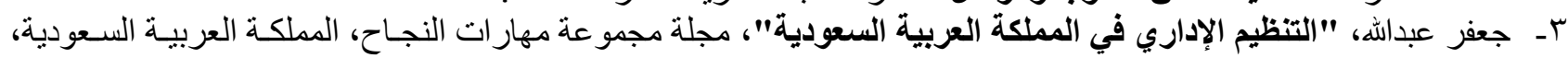
$r+11$

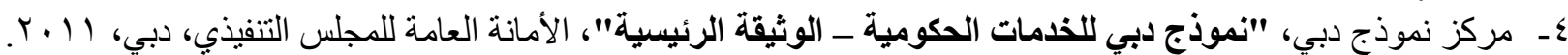

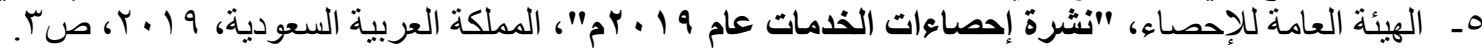

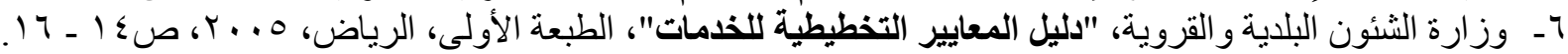


Vـ الهيئة العامة لتطوير مدينة الرياض، "الخطة التنفيذية لتسـيق وتـوفير الخدمات العامـة بمدينـة الريـاض"، المملكـة العربيـة

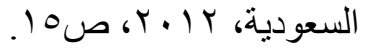

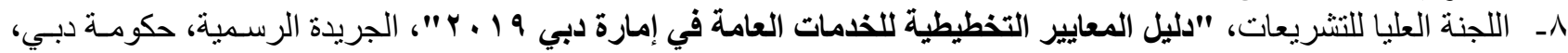

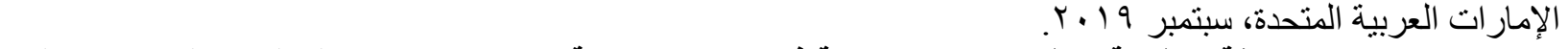

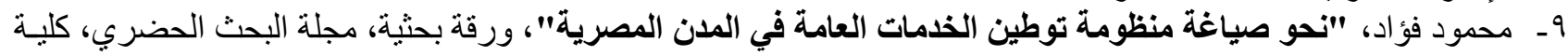

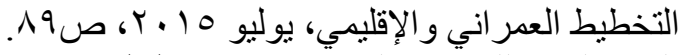

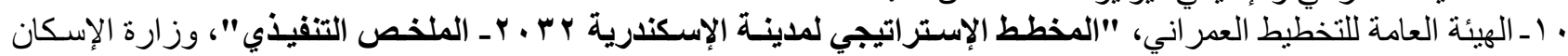

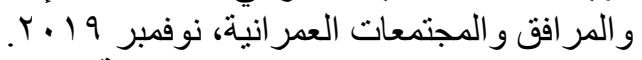

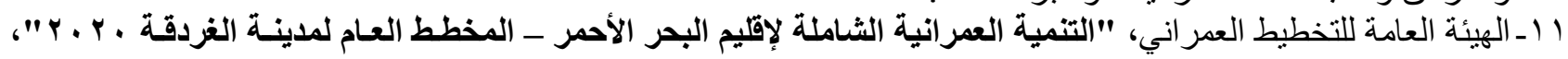

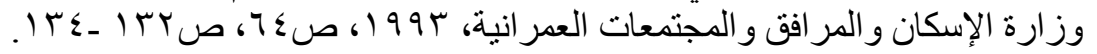

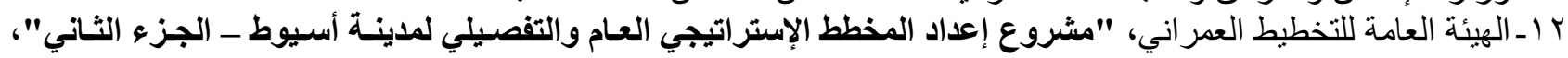

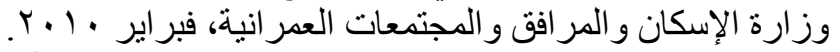

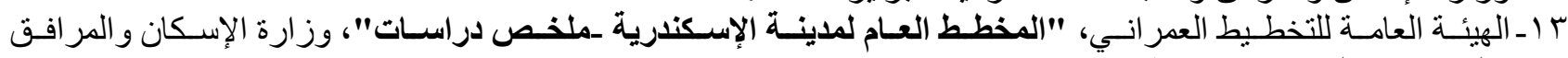

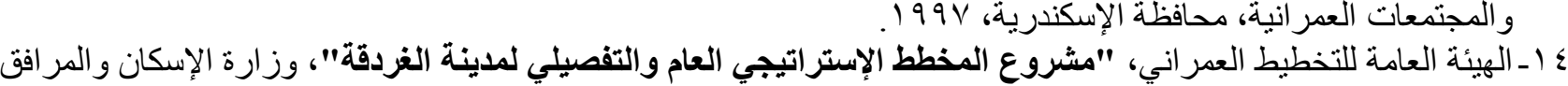

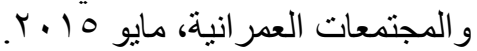

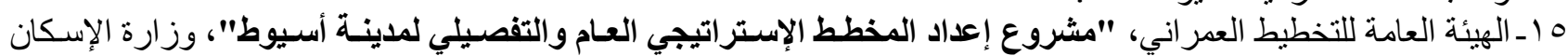

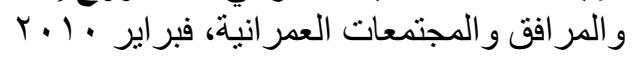

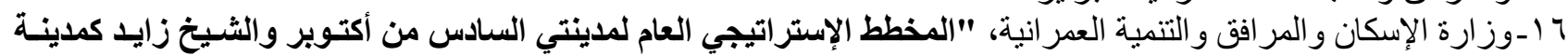

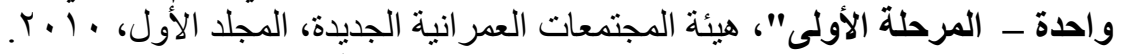

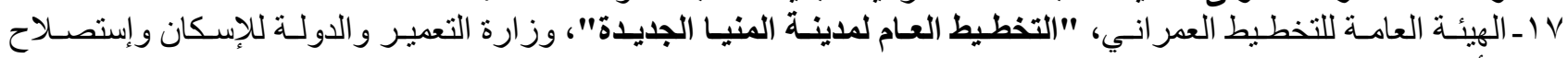

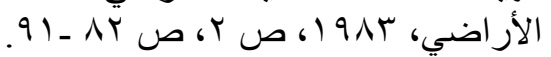

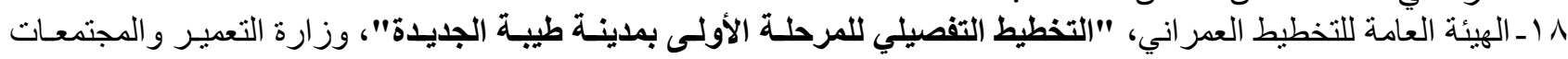

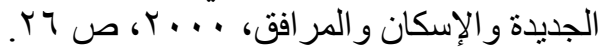

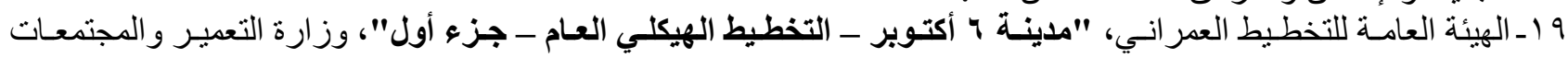

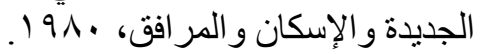

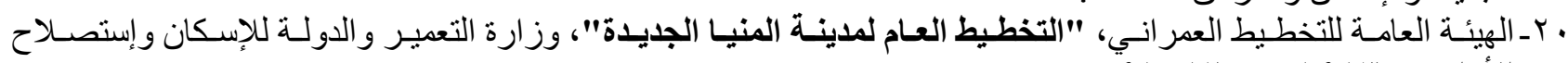

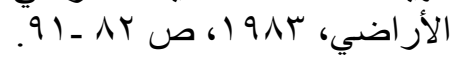

21-Bauby, Pierre \& Others, "Public Services in the European Union \& in the 27 Member States", The European Comission, 2010.

22-Adam, A, \& Others, "Guidelines for Human Settlement Planning and Design", CSIR Building and Construction Technology, Vol.1, Pretoria, 2005.

23-Benbabaali, Dalal, "Questioning the Role of the Indian Administrative Service in National Integration", South Asia Multidisciplinary Academic Journal, Paris, 2008, P3.

24- Planning Department, "Hong Kong Planning Standards and Guidelines - Ch.3: Community Facilities", The Government of the Hong Kong Special Administrative Region, December 2018.

25-Town and Country Planning Organization, "Urban and Regional Development Plans Formulation and Implementation Guidelines", Ministry of Urban Development, Government of India, 2015. 